\title{
The Biological Impact of Ionic Liquids Based on Sustainable Fatty Acid Anions Examined with a Tripartite Test System
}

Tobias Gundolf ${ }^{\dagger}$, Nadine Weyhing-Zerrer , Julia Sommer ${ }^{\dagger}$, Roland Kalb ${ }^{\ddagger}$, Dagmar Schoder , Peter Rossmanith ${ }^{\dagger}$, , Patrick Mester ${ }^{\dagger^{*}}$

$\uparrow$ Christian Doppler Laboratory for Monitoring of Microbial Contaminants, University of Veterinary Medicine, Veterinaerplatz 1, 1210 Vienna, Austria

* email address: Patrick-julian.mester@vetmeduni.ac.at

Merck KGaA, Life Science, Frankfurter Str. 250, 64293 Darmstadt, Germany

† Proionic GmbH, Parkring 18, 8074 Grambach, Austria

Institute of Food Safety, Food Technology and Veterinary Public Health, Department of Farm Animals and Public Health in Veterinary Medicine, University of Veterinary Medicine, Veterinaerplatz 1, 1210 Vienna, Austria

Total number of pages: 15

Total number of figures: 14

Total number of tables: 8 
Table S1. List of all commercial ILs used in this study including systematic names, abbreviations, chemical formulae, molar masses, nominal purities and suppliers.

\begin{tabular}{lccccc}
\hline \multicolumn{1}{c}{ Systematic name } & Abbreviation & Chemical formula & Molar Mass & Purity & Supplier \\
\hline 1-Ethyl-3-methylimidazolium chloride & {$\left[\mathrm{C}_{2}\right.$ mim $] \mathrm{Cl}$} & $\mathrm{C}_{6} \mathrm{H}_{11} \mathrm{ClN}_{2}$ & 146.62 & $>98 \%$ & Proionic GmbH \\
1-Butyl-3-methylimidazolium chloride & {$\left[\mathrm{C}_{4} \mathrm{mim}\right] \mathrm{Cl}$} & $\mathrm{C}_{8} \mathrm{H}_{15} \mathrm{ClN}_{2}$ & 174.67 & $>98 \%$ & Proionic GmbH \\
1-Hexyl-3-methylimidazolium chloride & {$\left[\mathrm{C}_{6} \mathrm{mim}\right] \mathrm{Cl}$} & $\mathrm{C}_{10} \mathrm{H}_{19} \mathrm{ClN}_{2}$ & 202.72 & $>98 \%$ & Proionic GmbH \\
1-Octyl-3-methylimidazolium chloride & {$\left[\mathrm{C}_{8} \mathrm{mim}\right] \mathrm{Cl}$} & $\mathrm{C}_{12} \mathrm{H}_{23} \mathrm{ClN}_{2}$ & 230.78 & $>98 \%$ & Proionic GmbH \\
1-Decyl-3-methylimidazolium chloride & {$\left[\mathrm{C}_{10}\right.$ mim $] \mathrm{Cl}$} & $\mathrm{C}_{14} \mathrm{H}_{27} \mathrm{ClN}_{2}$ & 258.83 & $>98 \%$ & Proionic GmbH \\
1-Dodecyl-3-methylimidazolium chloride & {$\left[\mathrm{C}_{12} \mathrm{mim}\right] \mathrm{Cl}$} & $\mathrm{C}_{16} \mathrm{H}_{31} \mathrm{ClN}_{2}$ & 286.89 & $>98 \%$ & Iolitec GmbH \\
1-Methyl-3-tetradecylimidazolium chloride & {$\left[\mathrm{C}_{14} \mathrm{mim}\right] \mathrm{Cl}$} & $\mathrm{C}_{18} \mathrm{H}_{35} \mathrm{ClN}_{2}$ & 314.94 & $>98 \%$ & Iolitec GmbH \\
1-Hexadecyl-3-methylimidazolium chloride & {$\left[\mathrm{C}_{16}\right.$ mim $] \mathrm{Cl}$} & $\mathrm{C}_{20} \mathrm{H}_{39} \mathrm{ClN}_{2}$ & 343 & $>98 \%$ & Iolitec GmbH \\
\hline
\end{tabular}

Table S2. List of all commercial fatty acids used in this study including systematic names, chemical formulae, molar masses, pKa-values, purities and supplier.

\begin{tabular}{lccccc}
\hline \multicolumn{1}{c}{ Systematic name } & Chemical formula & Molar Mass & pKa-value & Purity & Supplier \\
\hline Methanoic acid & $\mathrm{CH}_{2} \mathrm{O}_{2}$ & 46.03 & 3.77 & $95-97 \%$ & Sigma Alldrich \\
Ethanoic acid & $\mathrm{C}_{2} \mathrm{H}_{4} \mathrm{O}_{2}$ & 60.05 & 4.76 & $\geq 99.8 \%$ & Sigma Alldrich \\
Propionic acid & $\mathrm{C}_{3} \mathrm{H}_{6} \mathrm{O}_{2}$ & 74.08 & 4.88 & $99 \%$ & Sigma Alldrich \\
Butanoic acid & $\mathrm{C}_{4} \mathrm{H}_{8} \mathrm{O}_{2}$ & 88.11 & 4.82 & $\geq 99 \%$ & Sigma Alldrich \\
Hexanoic acid & $\mathrm{C}_{6} \mathrm{H}_{12} \mathrm{O}_{2}$ & 116.16 & 4.88 & $\geq 99 \%$ & Sigma Alldrich \\
Octanoic acid & $\mathrm{C}_{8} \mathrm{H}_{16} \mathrm{O}_{2}$ & 144.21 & 4.89 & $\geq 99 \%$ & Sigma Alldrich \\
Nonanoic acid & $\mathrm{C}_{9} \mathrm{H}_{18} \mathrm{O}_{2}$ & 158.24 & 4.96 & $\geq 97 \%$ & Sigma Alldrich \\
Decanoic acid & $\mathrm{C}_{10} \mathrm{H}_{20} \mathrm{O}_{2}$ & 172.27 & 4.9 & $\geq 98 \%$ & Sigma Alldrich \\
Dodecanoic acid & $\mathrm{C}_{12} \mathrm{H}_{24} \mathrm{O}_{9}$ & 200.32 & 5.3 & $\geq 98 \%$ & Sigma Alldrich \\
Tetradecanoic acid & $\mathrm{C}_{14} \mathrm{H}_{28} \mathrm{O}_{2}$ & 228.38 & 4.95 & $\geq 99.5 \%$ & Sigma Alldrich \\
Hexadecanoic acid & $\mathrm{C}_{16} \mathrm{H}_{32} \mathrm{O}_{2}$ & 256.43 & 4.95 & $\geq 99 \%$ & Sigma Alldrich \\
Octadecanoic acid & $\mathrm{C}_{18} \mathrm{H}_{36} \mathrm{O}_{2}$ & 284.48 & 4.95 & $\geq 98.5 \%$ & Sigma Alldrich \\
\hline
\end{tabular}


Table S3. List of all FAILs synthesized for this study including systematic names, abbreviations, chemical formulae and molar masses.

\begin{tabular}{|c|c|c|c|}
\hline Systematic name & Abbreviation & Chemical formula & Molar Mass \\
\hline 1-Ethyl-3-methylimidazolium methanoate & {$\left[\mathrm{C}_{2} \mathrm{mim}\right] \mathrm{CH}_{1} \mathrm{O}_{2}$} & $\mathrm{C}_{7} \mathrm{H}_{12} \mathrm{~N}_{2} \mathrm{O}_{2}$ & 156.18 \\
\hline 1-Ethyl-3-methylimidazolium ethanoate & {$\left[\mathrm{C}_{2} \operatorname{mim}\right] \mathrm{C}_{2} \mathrm{H}_{3} \mathrm{O}_{2}$} & $\mathrm{C}_{8} \mathrm{H}_{14} \mathrm{~N}_{2} \mathrm{O}_{2}$ & 170.21 \\
\hline 1-Ethyl-3-methylimidazolium propanoate & {$\left[\mathrm{C}_{2} \operatorname{mim}\right] \mathrm{C}_{3} \mathrm{H}_{5} \mathrm{O}_{2}$} & $\mathrm{C}_{9} \mathrm{H}_{16} \mathrm{~N}_{2} \mathrm{O}_{2}$ & 184.24 \\
\hline 1-Ethyl-3-methylimidazolium butanoate & {$\left[\mathrm{C}_{2} \operatorname{mim}\right] \mathrm{C}_{4} \mathrm{H}_{7} \mathrm{O}_{2}$} & $\mathrm{C}_{10} \mathrm{H}_{18} \mathrm{~N}_{2} \mathrm{O}_{2}$ & 198.26 \\
\hline 1-Ethyl-3-methylimidazolium hexanoate & {$\left[\mathrm{C}_{2} \operatorname{mim}\right] \mathrm{C}_{6} \mathrm{H}_{11} \mathrm{O}_{2}$} & $\mathrm{C}_{12} \mathrm{H}_{22} \mathrm{~N}_{2} \mathrm{O}_{2}$ & 226.32 \\
\hline 1-Ethyl-3-methylimidazolium octanoate & {$\left[\mathrm{C}_{2} \operatorname{mim}\right] \mathrm{C}_{8} \mathrm{H}_{15} \mathrm{O}_{2}$} & $\mathrm{C}_{14} \mathrm{H}_{26} \mathrm{~N}_{2} \mathrm{O}_{2}$ & 254.37 \\
\hline 1-Ethyl-3-methylimidazolium nonanoate & {$\left[\mathrm{C}_{2} \mathrm{mim}\right] \mathrm{C}_{9} \mathrm{H}_{17} \mathrm{O}_{2}$} & $\mathrm{C}_{15} \mathrm{H}_{28} \mathrm{~N}_{2} \mathrm{O}_{2}$ & 268.40 \\
\hline 1-Ethyl-3-methylimidazolium decanoate & {$\left[\mathrm{C}_{2} \operatorname{mim}\right] \mathrm{C}_{10} \mathrm{H}_{19} \mathrm{O}_{2}$} & $\mathrm{C}_{16} \mathrm{H}_{30} \mathrm{~N}_{2} \mathrm{O}_{2}$ & 282.42 \\
\hline 1-Ethyl-3-methylimidazolium dodecanoate & {$\left[\mathrm{C}_{2} \operatorname{mim}\right] \mathrm{C}_{12} \mathrm{H}_{23} \mathrm{O}_{9}$} & $\mathrm{C}_{18} \mathrm{H}_{34} \mathrm{~N}_{2} \mathrm{O}_{2}$ & 310.47 \\
\hline 1-Ethyl-3-methylimidazolium tetradecanoate & {$\left[\mathrm{C}_{2} \mathrm{mim}\right] \mathrm{C}_{14} \mathrm{H}_{27} \mathrm{O}_{2}$} & $\mathrm{C}_{20} \mathrm{H}_{38} \mathrm{~N}_{2} \mathrm{O}_{2}$ & 338.53 \\
\hline 1-Ethyl-3-methylimidazolium hexadecanoate & {$\left[\mathrm{C}_{2} \operatorname{mim}\right] \mathrm{C}_{16} \mathrm{H}_{31} \mathrm{O}_{2}$} & $\mathrm{C}_{22} \mathrm{H}_{42} \mathrm{~N}_{2} \mathrm{O}_{2}$ & 366.58 \\
\hline 1-Ethyl-3-methylimidazolium octadecanoate & {$\left[\mathrm{C}_{2} \mathrm{mim}\right] \mathrm{C}_{18} \mathrm{H}_{35} \mathrm{O}_{2}$} & $\mathrm{C}_{24} \mathrm{H}_{46} \mathrm{~N}_{2} \mathrm{O}_{2}$ & 394.63 \\
\hline 1-Ethyl-3-dimethylimidazolium methanoate & {$\left[\mathrm{C}_{2,1} \operatorname{mim}\right] \mathrm{CH}_{1} \mathrm{O}_{2}$} & $\mathrm{C}_{8} \mathrm{H}_{14} \mathrm{~N}_{2} \mathrm{O}_{2}$ & 170.21 \\
\hline 1-Ethyl-3-dimethylimidazolium ethanoate & {$\left[\mathrm{C}_{2,1} \mathrm{mim}\right] \mathrm{C}_{2} \mathrm{H}_{3} \mathrm{O}_{2}$} & $\mathrm{C}_{9} \mathrm{H}_{16} \mathrm{~N}_{2} \mathrm{O}_{2}$ & 184.24 \\
\hline 1-Ethyl-3-dimethylimidazolium propanoate & {$\left[\mathrm{C}_{2,1} \mathrm{mim}\right] \mathrm{C}_{3} \mathrm{H}_{5} \mathrm{O}_{2}$} & $\mathrm{C}_{10} \mathrm{H}_{18} \mathrm{~N}_{2} \mathrm{O}_{2}$ & 198.26 \\
\hline 1-Ethyl-3-dimethylimidazolium butanoate & {$\left[\mathrm{C}_{2,1} \mathrm{mim}\right] \mathrm{C}_{4} \mathrm{H}_{7} \mathrm{O}_{2}$} & $\mathrm{C}_{11} \mathrm{H}_{20} \mathrm{~N}_{2} \mathrm{O}_{2}$ & 212.29 \\
\hline 1-Ethyl-3-dimethylimidazolium hexanoate & {$\left[\mathrm{C}_{2,1} \operatorname{mim}\right] \mathrm{C}_{6} \mathrm{H}_{11} \mathrm{O}_{2}$} & $\mathrm{C}_{13} \mathrm{H}_{24} \mathrm{~N}_{2} \mathrm{O}_{2}$ & 240.34 \\
\hline 1-Ethyl-3-dimethylimidazolium octanoate & {$\left[\mathrm{C}_{2,1} \operatorname{mim}\right] \mathrm{C}_{8} \mathrm{H}_{15} \mathrm{O}_{2}$} & $\mathrm{C}_{15} \mathrm{H}_{28} \mathrm{~N}_{2} \mathrm{O}_{2}$ & 268.40 \\
\hline 1-Ethyl-3-dimethylimidazolium nonanoate & {$\left[\mathrm{C}_{2,1} \operatorname{mim}\right] \mathrm{C}_{9} \mathrm{H}_{17} \mathrm{O}_{2}$} & $\mathrm{C}_{16} \mathrm{H}_{30} \mathrm{~N}_{2} \mathrm{O}_{2}$ & 282.42 \\
\hline 1-Ethyl-3-dimethylimidazolium decanoate & {$\left[\mathrm{C}_{2,1} \operatorname{mim}\right] \mathrm{C}_{10} \mathrm{H}_{19} \mathrm{O}_{2}$} & $\mathrm{C}_{17} \mathrm{H}_{32} \mathrm{~N}_{2} \mathrm{O}_{2}$ & 296.45 \\
\hline 1-Ethyl-3-dimethylimidazolium dodecanoate & {$\left[\mathrm{C}_{2,1} \operatorname{mim}\right] \mathrm{C}_{12} \mathrm{H}_{23} \mathrm{O}_{9}$} & $\mathrm{C}_{19} \mathrm{H}_{36} \mathrm{~N}_{2} \mathrm{O}_{2}$ & 324.50 \\
\hline 1-Ethyl-3-dimethylimidazolium tetradecanoate & {$\left[\mathrm{C}_{2,1} \mathrm{mim}\right] \mathrm{C}_{14} \mathrm{H}_{27} \mathrm{O}_{2}$} & $\mathrm{C}_{21} \mathrm{H}_{40} \mathrm{~N}_{2} \mathrm{O}_{2}$ & 352.55 \\
\hline 1-Ethyl-3-dimethylimidazolium hexadecanoate & {$\left[\mathrm{C}_{2,1} \operatorname{mim}\right] \mathrm{C}_{16} \mathrm{H}_{31} \mathrm{O}_{2}$} & $\mathrm{C}_{23} \mathrm{H}_{44} \mathrm{~N}_{2} \mathrm{O}_{2}$ & 380.61 \\
\hline 1-Ethyl-3-dimethylimidazolium octadecanoate & {$\left[\mathrm{C}_{2,1} \mathrm{mim}\right] \mathrm{C}_{18} \mathrm{H}_{35} \mathrm{O}_{2}$} & $\mathrm{C}_{25} \mathrm{H}_{48} \mathrm{~N}_{2} \mathrm{O}_{2}$ & 408.66 \\
\hline 1-Ethyl-1-methylpiperidinium methanoate & {$\left[\mathrm{C}_{2,1} \mathrm{Pip}\right] \mathrm{CH}_{1} \mathrm{O}_{2}$} & $\mathrm{C}_{9} \mathrm{H}_{19} \mathrm{NO}_{2}$ & 173.25 \\
\hline 1-Ethyl-1-methylpiperidinium ethanoate & {$\left[\mathrm{C}_{2,1} \mathrm{Pip}\right] \mathrm{C}_{2} \mathrm{H}_{3} \mathrm{O}_{2}$} & $\mathrm{C}_{10} \mathrm{H}_{21} \mathrm{NO}_{2}$ & 187.28 \\
\hline 1-Ethyl-1-methylpiperidinium propanoate & {$\left[\mathrm{C}_{2,1} \mathrm{Pip}\right] \mathrm{C}_{3} \mathrm{H}_{5} \mathrm{O}_{2}$} & $\mathrm{C}_{11} \mathrm{H}_{23} \mathrm{NO}_{2}$ & 201.31 \\
\hline 1-Ethyl-1-methylpiperidinium butanoate & {$\left[\mathrm{C}_{2,1} \mathrm{Pip}\right] \mathrm{C}_{4} \mathrm{H}_{7} \mathrm{O}_{2}$} & $\mathrm{C}_{12} \mathrm{H}_{25} \mathrm{NO}_{2}$ & 215.33 \\
\hline 1-Ethyl-1-methylpiperidinium hexanoate & {$\left[\mathrm{C}_{2,1} \mathrm{Pip}\right] \mathrm{C}_{6} \mathrm{H}_{11} \mathrm{O}_{2}$} & $\mathrm{C}_{14} \mathrm{H}_{29} \mathrm{NO}_{2}$ & 243.39 \\
\hline 1-Ethyl-1-methylpiperidinium octanoate & {$\left[\mathrm{C}_{2,1} \mathrm{Pip}\right] \mathrm{C}_{8} \mathrm{H}_{15} \mathrm{O}_{2}$} & $\mathrm{C}_{16} \mathrm{H}_{33} \mathrm{NO}_{2}$ & 271.44 \\
\hline 1-Ethyl-1-methylpiperidinium nonanoate & {$\left[\mathrm{C}_{2,1} \mathrm{Pip}\right] \mathrm{C}_{9} \mathrm{H}_{17} \mathrm{O}_{2}$} & $\mathrm{C}_{17} \mathrm{H}_{35} \mathrm{NO}_{2}$ & 285.47 \\
\hline 1-Ethyl-1-methylpiperidinium decanoate & {$\left[\mathrm{C}_{2,1} \mathrm{Pip}\right] \mathrm{C}_{10} \mathrm{H}_{19} \mathrm{O}_{2}$} & $\mathrm{C}_{18} \mathrm{H}_{37} \mathrm{NO}_{2}$ & 299.49 \\
\hline 1-Ethyl-1-methylpiperidinium dodecanoate & {$\left[\mathrm{C}_{2,1} \mathrm{Pip}\right] \mathrm{C}_{12} \mathrm{H}_{23} \mathrm{O}_{9}$} & $\mathrm{C}_{20} \mathrm{H}_{41} \mathrm{NO}_{2}$ & 327.55 \\
\hline 1-Ethyl-1-methylpiperidinium tetradecanoate & {$\left[\mathrm{C}_{2,1} \mathrm{Pip}\right] \mathrm{C}_{14} \mathrm{H}_{27} \mathrm{O}_{2}$} & $\mathrm{C}_{22} \mathrm{H}_{44} \mathrm{NO}_{2}$ & 355.60 \\
\hline 1-Ethyl-1-methylpiperidinium hexadecanoate & {$\left[\mathrm{C}_{2,1} \mathrm{Pip}\right] \mathrm{C}_{16} \mathrm{H}_{31} \mathrm{O}_{2}$} & $\mathrm{C}_{24} \mathrm{H}_{49} \mathrm{NO}_{2}$ & 383.65 \\
\hline 1-Ethyl-1-methylpiperidinium octadecanoate & {$\left[\mathrm{C}_{2,1} \mathrm{Pip}\right] \mathrm{C}_{18} \mathrm{H}_{35} \mathrm{O}_{2}$} & $\mathrm{C}_{26} \mathrm{H}_{53} \mathrm{NO}_{2}$ & 411.70 \\
\hline 4-Ethyl-4-methylmorpholinium methanoate & {$\left[\mathrm{C}_{2,1}\right.$ mor $] \mathrm{CH}_{1} \mathrm{O}_{2}$} & $\mathrm{C}_{8} \mathrm{H}_{17} \mathrm{NO}_{3}$ & 175.23 \\
\hline 4-Ethyl-4-methylmorpholinium ethanoate & {$\left[\mathrm{C}_{2,1}\right.$ mor $] \mathrm{C}_{2} \mathrm{H}_{3} \mathrm{O}_{2}$} & $\mathrm{C}_{9} \mathrm{H}_{19} \mathrm{NO}_{3}$ & 189.25 \\
\hline 4-Ethyl-4-methylmorpholinium propanoate & {$\left[\mathrm{C}_{2,1}\right.$ mor $] \mathrm{C}_{3} \mathrm{H}_{5} \mathrm{O}_{2}$} & $\mathrm{C}_{10} \mathrm{H}_{21} \mathrm{NO}_{3}$ & 203.28 \\
\hline 4-Ethyl-4-methylmorpholinium butanoate & {$\left[\mathrm{C}_{2,1}\right.$ mor $] \mathrm{C}_{4} \mathrm{H}_{7} \mathrm{O}_{2}$} & $\mathrm{C}_{11} \mathrm{H}_{23} \mathrm{NO}_{3}$ & 217.30 \\
\hline 4-Ethyl-4-methylmorpholinium hexanoate & {$\left[\mathrm{C}_{2,1}\right.$ mor $] \mathrm{C}_{6} \mathrm{H}_{11} \mathrm{O}_{2}$} & $\mathrm{C}_{13} \mathrm{H}_{27} \mathrm{NO}_{3}$ & 245.36 \\
\hline 4-Ethyl-4-methylmorpholinium octanoate & {$\left[\mathrm{C}_{2,1}\right.$ mor $] \mathrm{C}_{8} \mathrm{H}_{15} \mathrm{O}_{2}$} & $\mathrm{C}_{15} \mathrm{H}_{31} \mathrm{NO}_{3}$ & 273.41 \\
\hline 4-Ethyl-4-methylmorpholinium nonanoate & {$\left[\mathrm{C}_{2,1}\right.$ mor $] \mathrm{C}_{9} \mathrm{H}_{17} \mathrm{O}_{2}$} & $\mathrm{C}_{16} \mathrm{H}_{33} \mathrm{NO}_{3}$ & 287.44 \\
\hline 4-Ethyl-4-methylmorpholinium decanoate & {$\left[\mathrm{C}_{2,1} \mathrm{mor}\right] \mathrm{C}_{10} \mathrm{H}_{19} \mathrm{O}_{2}$} & $\mathrm{C}_{17} \mathrm{H}_{35} \mathrm{NO}_{3}$ & 301.46 \\
\hline 4-Ethyl-4-methylmorpholinium dodecanoate & {$\left[\mathrm{C}_{2,1} \mathrm{mor}\right] \mathrm{C}_{12} \mathrm{H}_{23} \mathrm{O}_{9}$} & $\mathrm{C}_{19} \mathrm{H}_{39} \mathrm{NO}_{3}$ & 329.52 \\
\hline 4-Ethyl-4-methylmorpholinium tetradecanoate & {$\left[\mathrm{C}_{2,1} \mathrm{mor}\right] \mathrm{C}_{14} \mathrm{H}_{27} \mathrm{O}_{2}$} & $\mathrm{C}_{21} \mathrm{H}_{43} \mathrm{NO}_{3}$ & 357.57 \\
\hline 4-Ethyl-4-methylmorpholinium hexadecanoate & {$\left[\mathrm{C}_{2,1}\right.$ mor $] \mathrm{C}_{16} \mathrm{H}_{31} \mathrm{O}_{2}$} & $\mathrm{C}_{23} \mathrm{H}_{47} \mathrm{NO}_{3}$ & 385.62 \\
\hline 4-Ethyl-4-methylmorpholinium octadecanoate & {$\left[\mathrm{C}_{2,1} \mathrm{mor}\right] \mathrm{C}_{18} \mathrm{H}_{35} \mathrm{O}_{2}$} & $\mathrm{C}_{25} \mathrm{H}_{51} \mathrm{NO}_{3}$ & 413.68 \\
\hline
\end{tabular}




\section{NMR Spectra}

In connection with the interpretation of the NMR spectra, we have to point out, that FAILs with 1,3-dialkylimidazolium cations always form more or less 1,3-dialkylimidazolium-2carboxylates, whenever they come in contact with air. There is always some formation of carbenes via deprotonation of the imidazolium-H2-ring proton by the basic anion, which subsequently reacts with $\mathrm{CO}_{2}$ forming the 2-carboxylate (see e.g. Gabriela Gurau, Hector Rodriguez, Steven P. Kelley, Peter Janiczek, Roland S. Kalb and Robin D. Rogers; Angew. Chem. Int. Ed. 2011, 50, 12024 -12026). This 2-carboxylate reacts reversibly on addition of water, forming back the pure ionic liquid quickly. As the hydrolysis of the 2-carboxylates in excess water proceeds quickly (minutes at room temperature) and quantitatively, we can be pretty sure, that we did have 2-carboxylate free 1,3-dialkylimidazolium ionic liquids in the present study as soon as the ionic liquids were diluted with water. On the other side, should theoretically not all 2-carboxylates be hydrolyzed in water, e.g. long chain FAIL 2carboxylates not investigated so far, they would be an intrinsic, unavoidable impurity like e.g. carbonates in sodium hydroxide. Signals at $\delta=3.16 \mathrm{ppm}$ belong to traces of residual methanol, used as a solvent in synthesis of the FAILs .

Figure S1. ${ }^{1} \mathrm{H}$ NMR spectrum of $\left[\mathrm{C}_{2} \mathrm{mim}\right] \mathrm{CH}_{1} \mathrm{O}_{2}$. (300MHz, d6-DMSO)

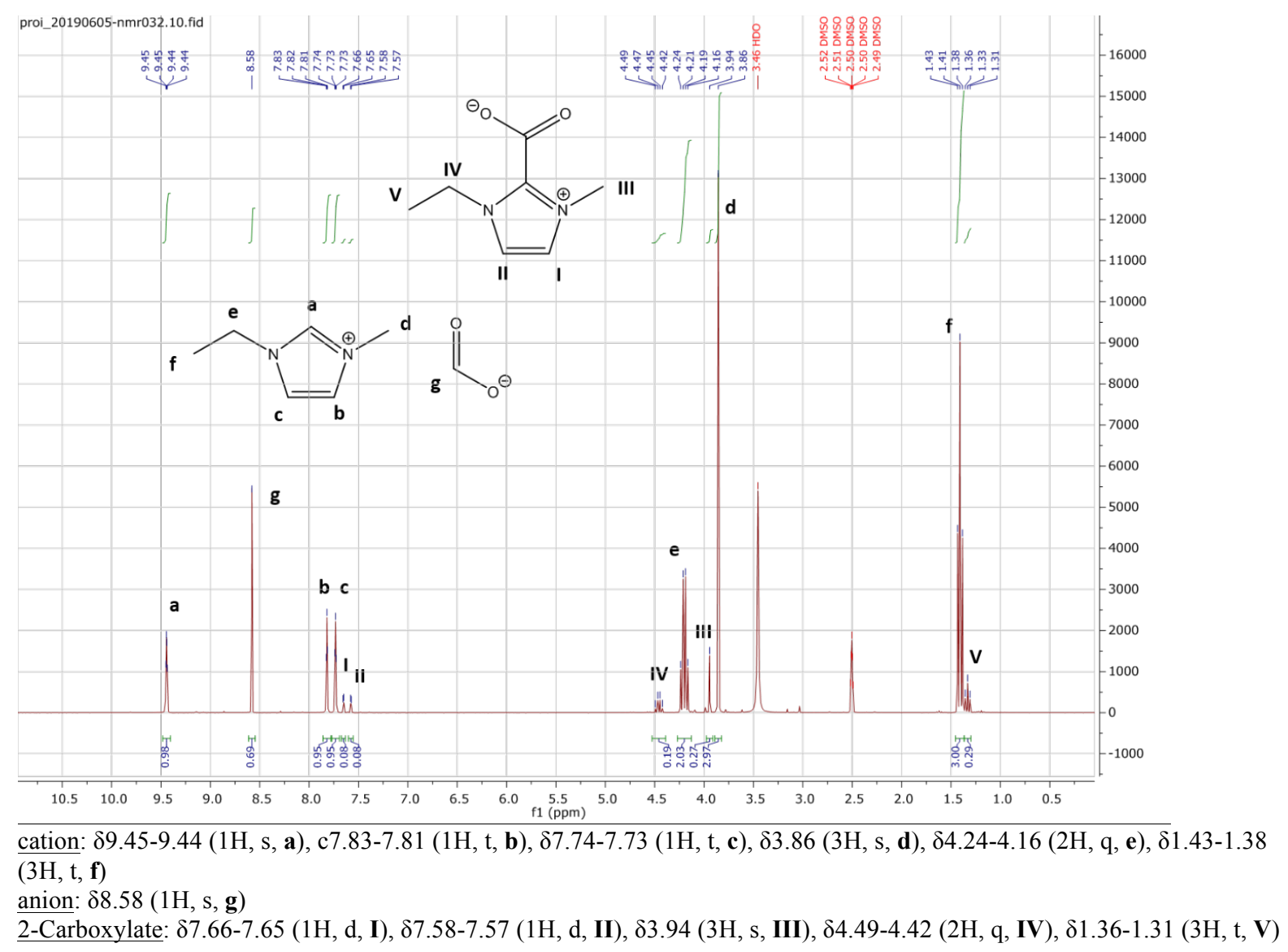


Figure S2. ${ }^{1} \mathrm{H}$ NMR spectrum of $\left[\mathrm{C}_{2} \mathrm{mim}\right] \mathrm{C}_{2} \mathrm{H}_{3} \mathrm{O}_{2}$. (300MHz, d6-DMSO)

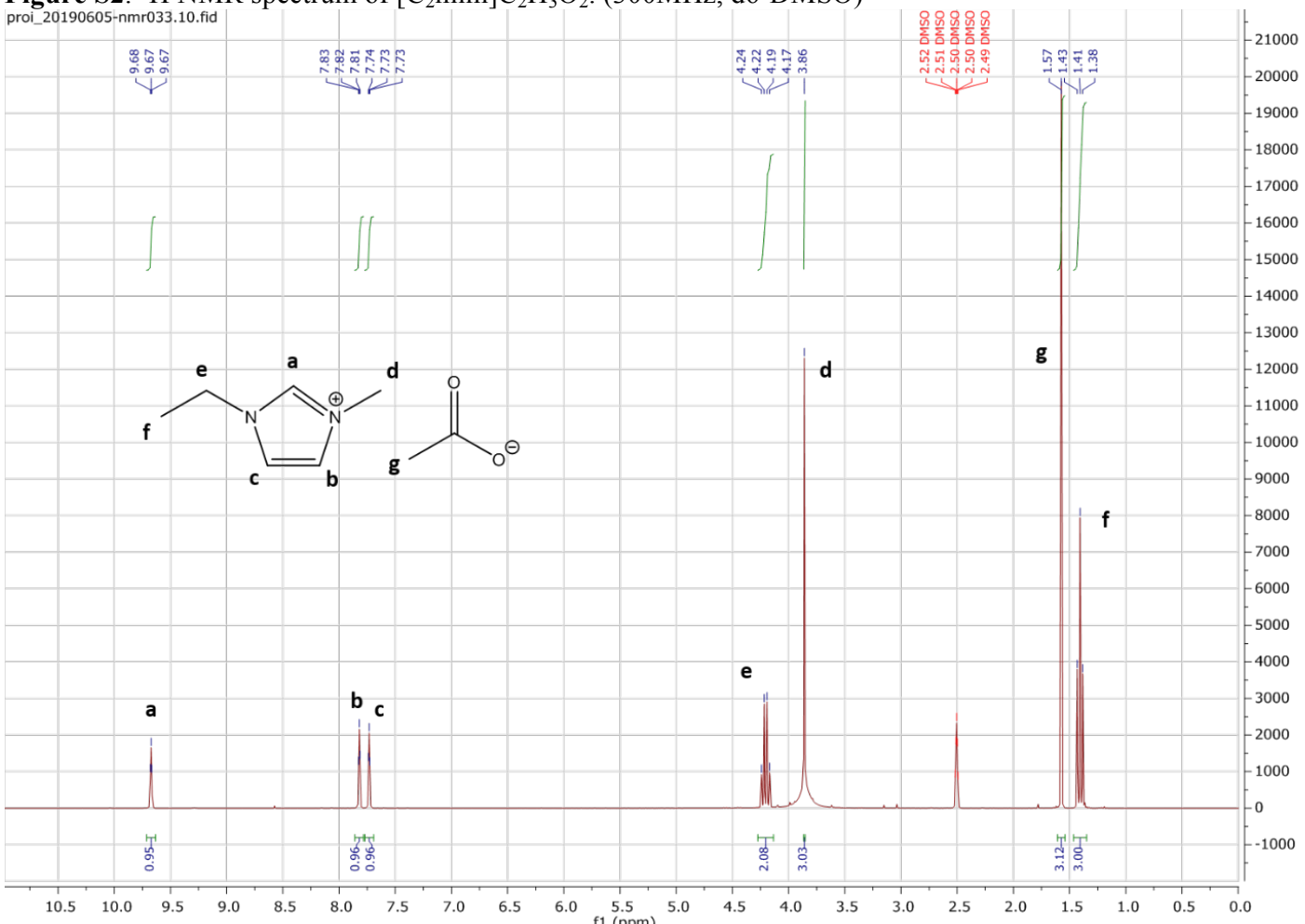

cation: $\delta 9.68-9.67(1 \mathrm{H}, \mathrm{s}, \mathbf{a}), \mathrm{c} 7.83-7.81(1 \mathrm{H}, \mathrm{t}, \mathbf{b}), \delta 7.74-7.73(1 \mathrm{H}, \mathrm{t}, \mathbf{c}), \delta 3.86(3 \mathrm{H}, \mathrm{s}, \mathbf{d}), \delta 4.24-4.17(2 \mathrm{H}, \mathrm{q}, \mathbf{e}), \delta 1.43-1.38$ $(3 \mathrm{H}, \mathrm{t}, \mathbf{f})$

anion: $\delta 1.57(3 \mathrm{H}, \mathrm{s}, \mathbf{g})$

Figure S3. ${ }^{1} \mathrm{H}$ NMR spectrum of $\left[\mathrm{C}_{2} \mathrm{mim}\right] \mathrm{C}_{3} \mathrm{H}_{5} \mathrm{O}_{2}$. (300MHz, d6-DMSO)

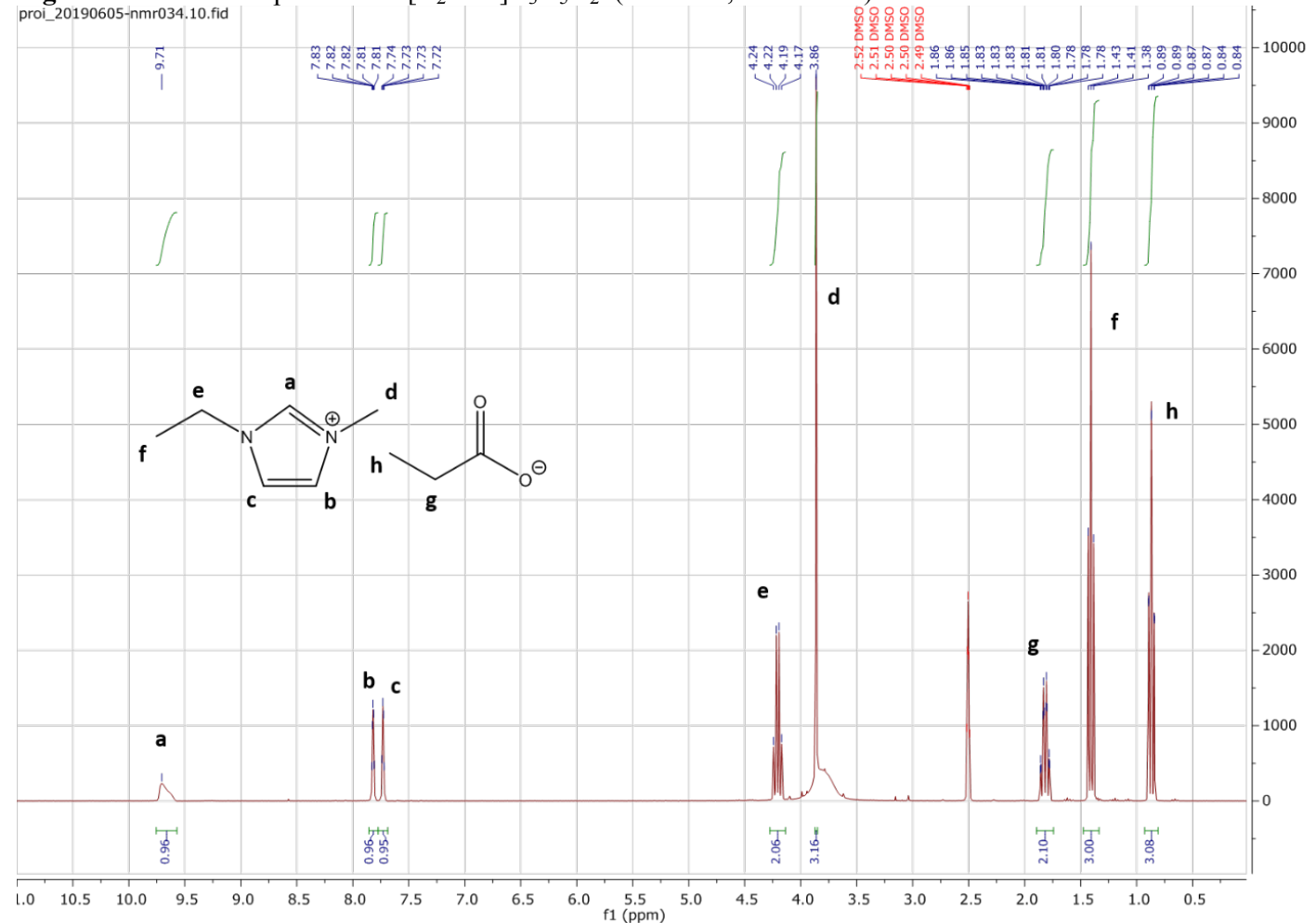

cation: $\delta 9.71(1 \mathrm{H}, \mathrm{s}, \mathbf{a}), \delta 7.83-7.81(1 \mathrm{H}, \mathrm{t}, \mathbf{b}), \delta 7.74-7.72(1 \mathrm{H}, \mathrm{t}, \mathbf{c}), \delta 3.86(3 \mathrm{H}, \mathrm{s}, \mathbf{d}), \delta 4.24-4.17(2 \mathrm{H}, \mathrm{q}, \mathbf{e}), \delta 1.43-1.38(3 \mathrm{H}, \mathrm{t}$,

anion: $\delta 1.86-1.78(2 \mathrm{H}, \mathrm{q}, \mathbf{g}), \delta 0.89-0.84(3 \mathrm{H}, \mathrm{t}, \mathbf{h})$ 
Figure S4. ${ }^{1} \mathrm{H}$ NMR spectrum of $\left[\mathrm{C}_{2} \mathrm{mim}\right] \mathrm{C}_{4} \mathrm{H}_{7} \mathrm{O}_{2}$. (300MHz, d6-DMSO)

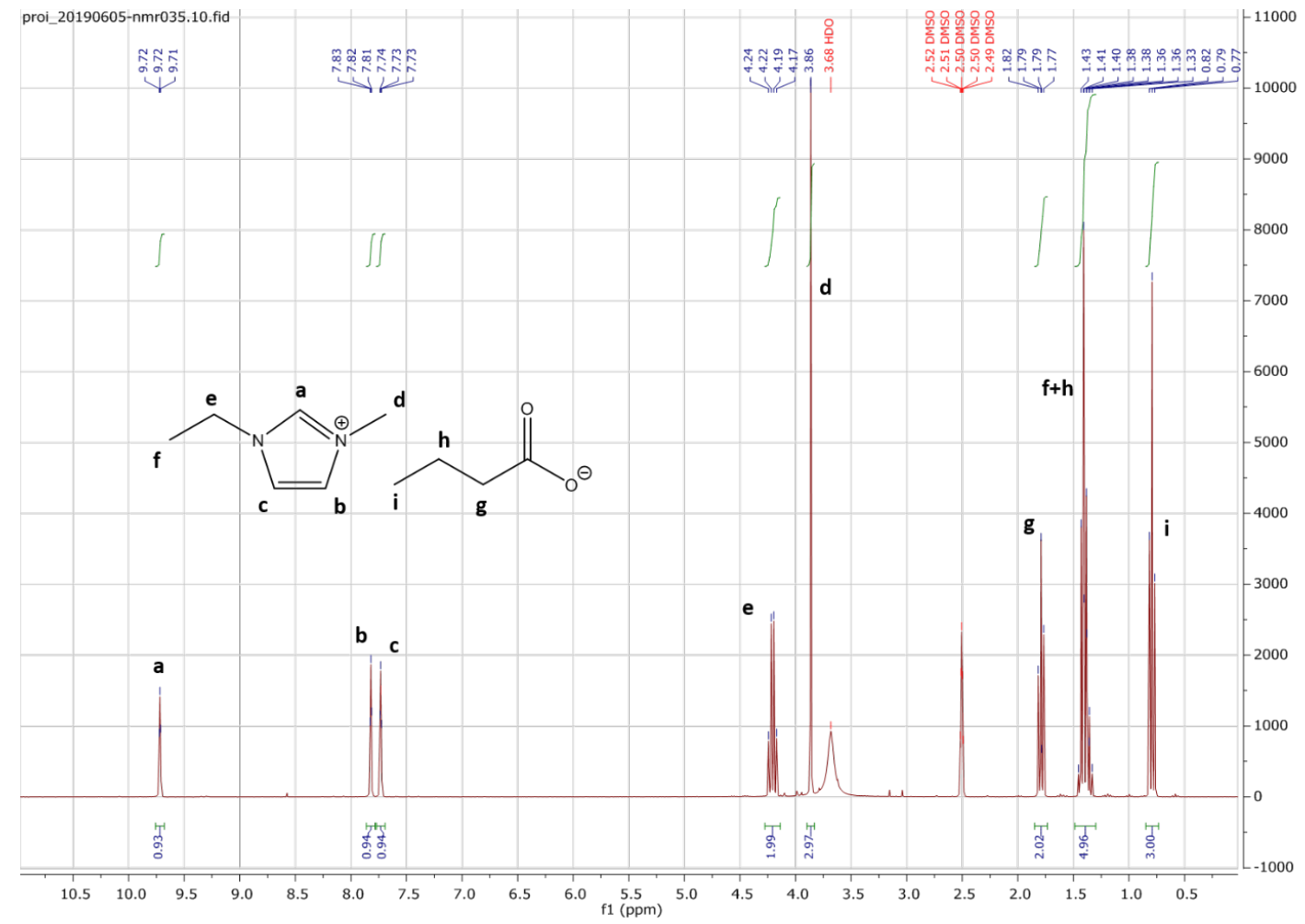

cation: $\delta 9.72-9.71(1 \mathrm{H}, \mathrm{s}, \mathbf{a}), \delta 7.83-7.81(1 \mathrm{H}, \mathrm{t}, \mathbf{b}), \delta 7.74-7.73(1 \mathrm{H}, \mathrm{t}, \mathbf{c}), \delta 3.86(3 \mathrm{H}, \mathrm{s}, \mathbf{d}), \delta 4.24-4.17(2 \mathrm{H}, \mathrm{q}, \mathbf{e}), \delta 1.43-1.33$ $(3 \mathrm{H}, \mathrm{m}, \mathbf{f})$

anion: $\delta 1.82-1.77(2 \mathrm{H}, \mathrm{t}, \mathbf{g}), \delta 1.43-1.33(2 \mathrm{H}, \mathrm{m}, \mathbf{h}) \delta 0.82-0.77(3 \mathrm{H}, \mathrm{t}, \mathbf{i})$

Figure S5. ${ }^{1} \mathrm{H}$ NMR spectrum of $\left[\mathrm{C}_{2} \mathrm{mim}\right] \mathrm{C}_{6} \mathrm{H}_{11} \mathrm{O}_{2}$. (300MHz, d6-DMSO)

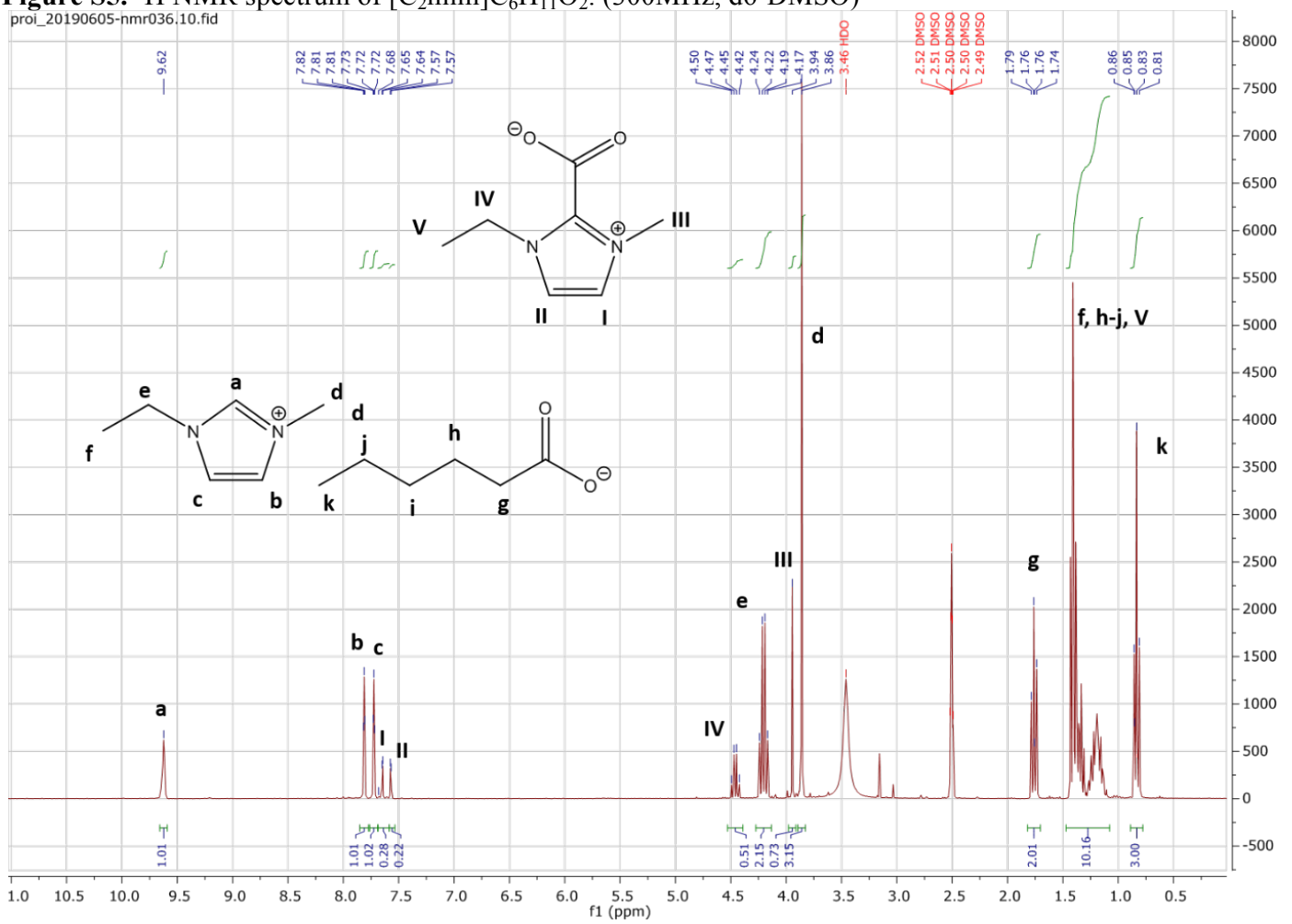

cation: $\delta 9.62(1 \mathrm{H}, \mathrm{s}, \mathbf{a}), \delta 7.82-7.81(1 \mathrm{H}, \mathrm{t}, \mathbf{b}), \delta 7.73-7.72(1 \mathrm{H}, \mathrm{t}, \mathbf{c}), \delta 3.86(3 \mathrm{H}, \mathrm{s}, \mathbf{d}), \delta 4.24-4.17(2 \mathrm{H}, \mathrm{q}, \mathbf{e}), \delta 1.43-1.11(3 \mathrm{H}$, $\mathrm{m}, \mathbf{f})$

anion: $\delta 1.79-1.74(2 \mathrm{H}, \mathrm{t}, \mathbf{g}), \delta 1.43-1.11(6 \mathrm{H}, \mathrm{m}, \mathbf{h}, \mathbf{i}, \mathbf{j}), \delta 0.86-0.83(3 \mathrm{H}, \mathrm{t}, \mathbf{k})$

2-Carboxylate: $\delta 7.68-7.64(1 \mathrm{H}, \mathrm{d}, \mathbf{I}), \delta 7.57(1 \mathrm{H}, \mathrm{d}, \mathbf{I I}), \delta 3.94(3 \mathrm{H}, \mathrm{s}, \mathbf{I I I}), \delta 4.50-4.42(2 \mathrm{H}, \mathrm{q}, \mathbf{I V}), \delta 1.43-1.11(3 \mathrm{H}, \mathrm{m}, \mathbf{V})$ 
Figure S6. ${ }^{1} \mathrm{H}$ NMR spectrum of $\left[\mathrm{C}_{2} \mathrm{mim}\right] \mathrm{C}_{8} \mathrm{H}_{15} \mathrm{O}_{2}$. (300MHz, d6-DMSO)

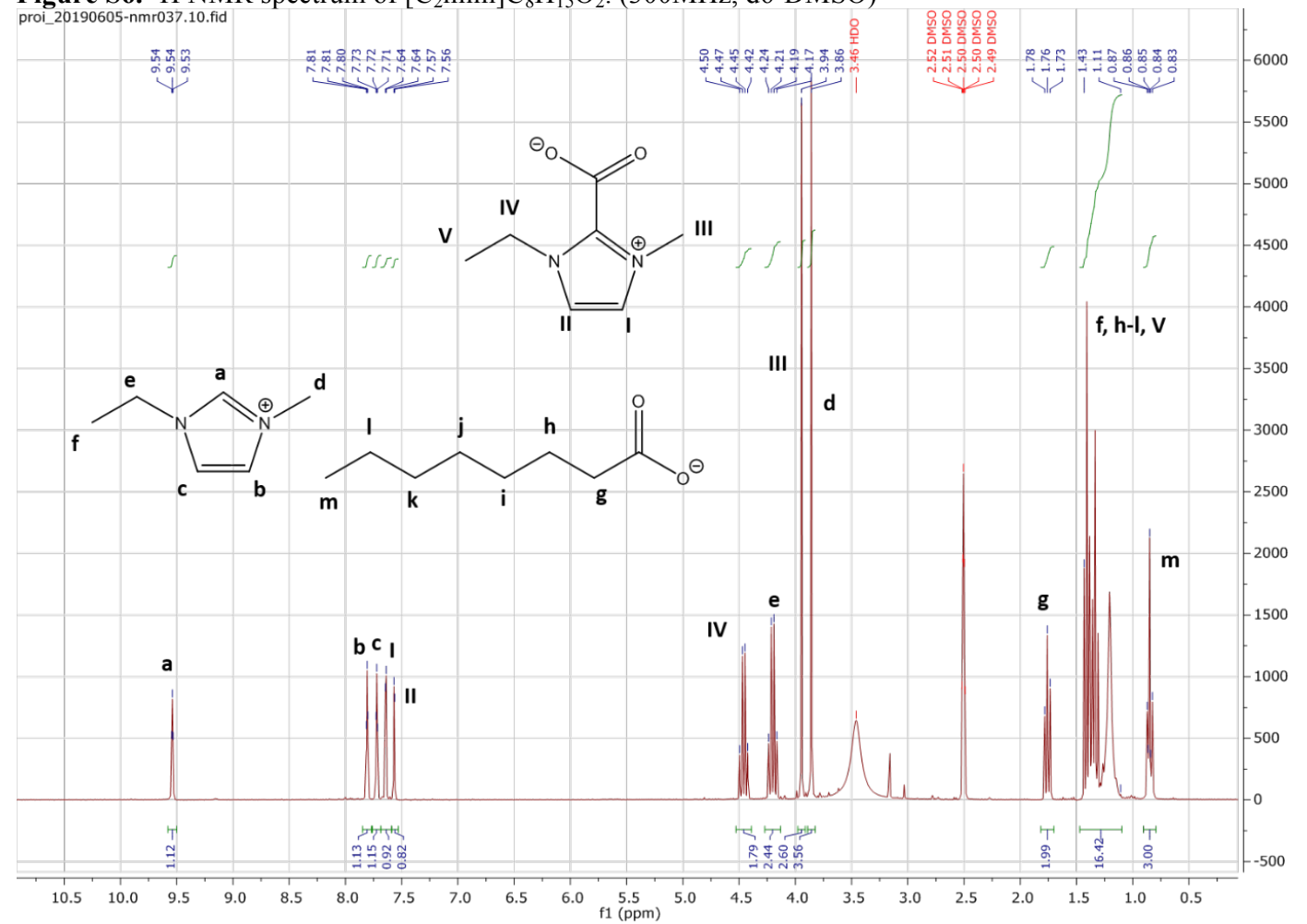

cation: $\delta 9.54-9.53(1 \mathrm{H}, \mathrm{s}, \mathbf{a}), \delta 7.81-7.80(1 \mathrm{H}, \mathrm{t}, \mathbf{b}), \delta 7.73-7.71(1 \mathrm{H}, \mathrm{t}, \mathbf{c}), \delta 3.86(3 \mathrm{H}, \mathrm{s}, \mathbf{d}), \delta 4.24-4.17(2 \mathrm{H}, \mathrm{q}, \mathbf{e}), \delta 1.43-1.11$ $(3 \mathrm{H}, \mathrm{m}, \mathbf{f})$

anion: $\delta 1.78-1.73(2 \mathrm{H}, \mathrm{t}, \mathbf{g}), \delta 1.43-1.11(10 \mathrm{H}, \mathrm{m}, \mathbf{h}, \mathbf{i}, \mathbf{j}, \mathbf{k}, \mathbf{l}), \delta 0.86-0.83(3 \mathrm{H}, \mathrm{t}, \mathbf{m})$

2-Carboxylate: $\delta 7.64(1 \mathrm{H}, \mathrm{d}, \mathbf{I}), \delta 7.57-7.56(1 \mathrm{H}, \mathrm{d}, \mathbf{I I}), \delta 3.94(3 \mathrm{H}, \mathrm{s}, \mathbf{I I I}), \delta 4.50-4.42(2 \mathrm{H}, \mathrm{q}, \mathbf{I V}), \delta 1.43-1.11(3 \mathrm{H}, \mathrm{m}, \mathbf{V})$

Figure S7. ${ }^{1} \mathrm{H}$ NMR spectrum of $\left[\mathrm{C}_{2} \mathrm{mim}\right] \mathrm{C}_{9} \mathrm{H}_{17} \mathrm{O}_{2}$. (300MHz, d6-DMSO)

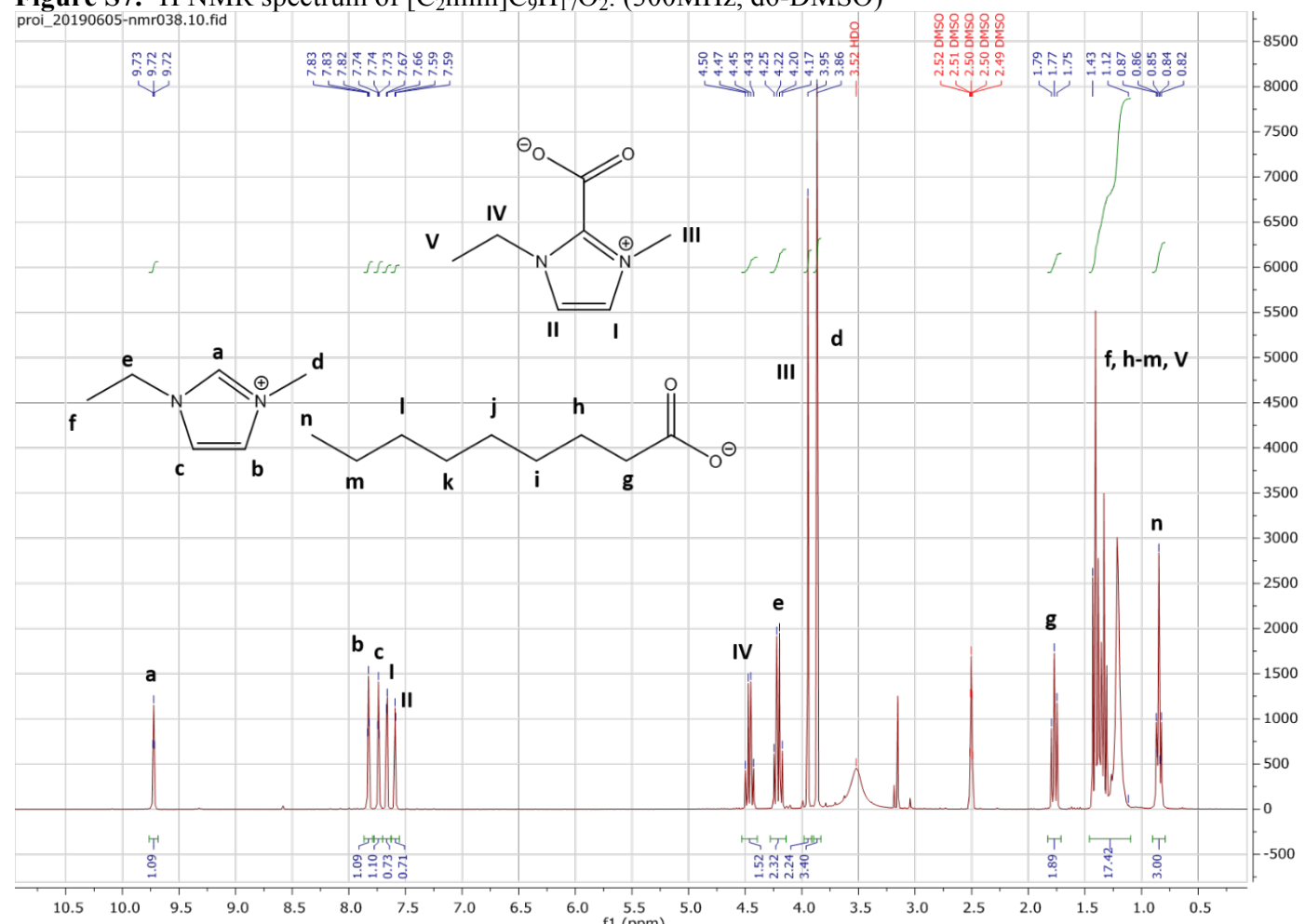

cation: $\delta 9.73-9.72(1 \mathrm{H}, \mathrm{s}, \mathbf{a}), \delta 7.83-7.82(1 \mathrm{H}, \mathrm{t}, \mathbf{b}), \delta 7.74-7.73(1 \mathrm{H}, \mathrm{t}, \mathbf{c}), \delta 3.86(3 \mathrm{H}, \mathrm{s}, \mathbf{d}), \delta 4.25-4.17(2 \mathrm{H}, \mathrm{q}, \mathbf{e}), \delta 1.43-1.12$ $(3 \mathrm{H}, \mathrm{m}, \mathbf{f})$

anion: $\delta 1.79-1.75(2 \mathrm{H}, \mathrm{t}, \mathbf{g}), \delta 1.43-1.12(12 \mathrm{H}, \mathrm{m}, \mathbf{h}, \mathbf{i}, \mathbf{j}, \mathbf{k}, \mathbf{l}, \mathbf{m}), \delta 0.87-0.82(3 \mathrm{H}, \mathrm{t}, \mathbf{n})$

2-Carboxylate: $\delta 7.67-7.66(1 \mathrm{H}, \mathrm{d}, \mathbf{I}), \delta 7.59(1 \mathrm{H}, \mathrm{d}, \mathbf{I I}), \delta 3.95(3 \mathrm{H}, \mathrm{s}, \mathbf{I I I}), \delta 4.50-4.43(2 \mathrm{H}, \mathrm{q}, \mathbf{I V}), \delta 1.43-1.12(3 \mathrm{H}, \mathrm{m}, \mathbf{V})$ 
Figure S8. ${ }^{1} \mathrm{H}$ NMR spectrum of $\left[\mathrm{C}_{2} \mathrm{mim}\right] \mathrm{C}_{10} \mathrm{H}_{19} \mathrm{O}_{2}$. (300MHz, d6-DMSO)

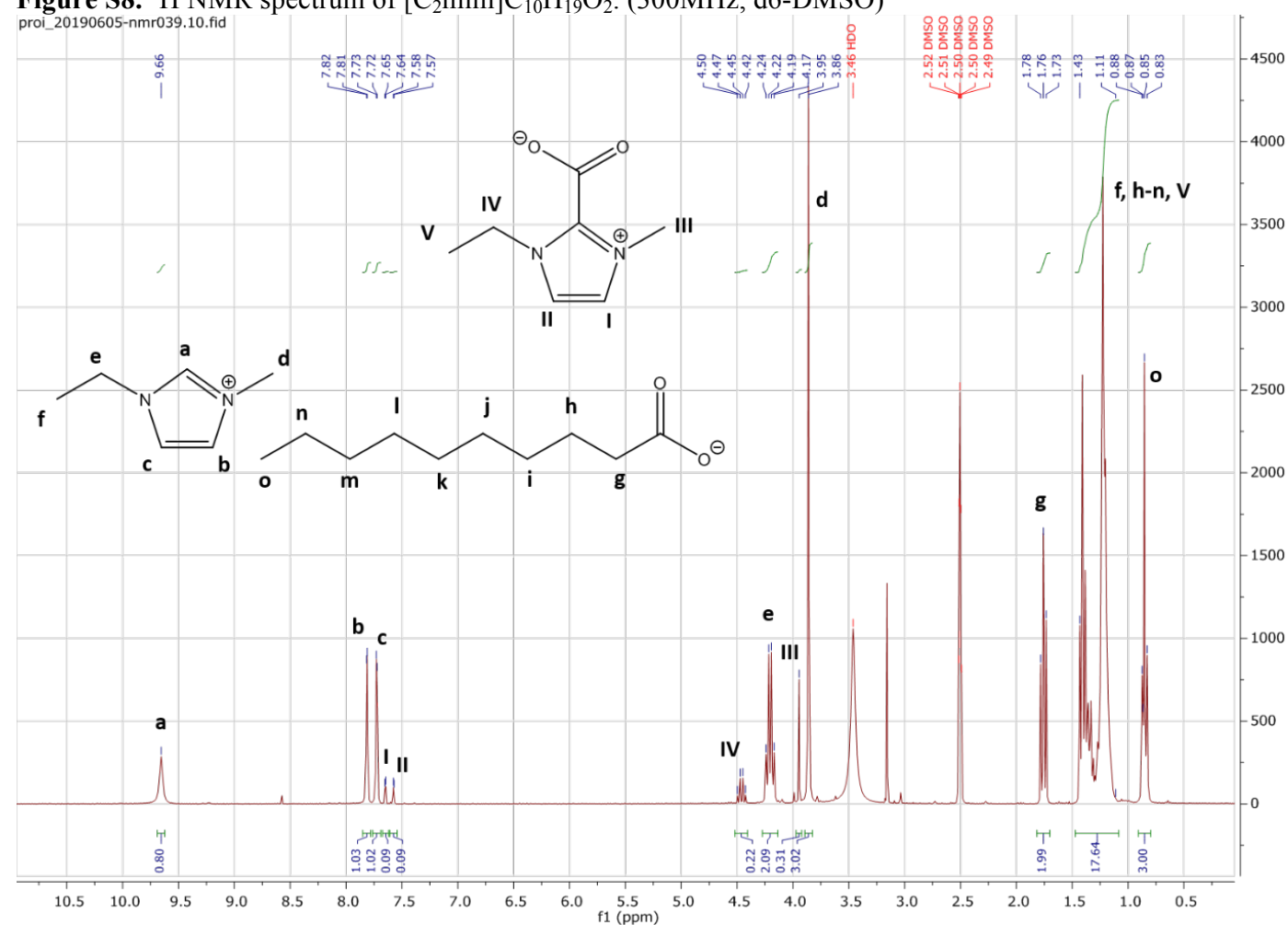

cation: $\delta 9.66(1 \mathrm{H}, \mathrm{s}, \mathbf{a}), \delta 7.82-7.81(1 \mathrm{H}, \mathrm{t}, \mathbf{b}), \delta 7.73-7.72(1 \mathrm{H}, \mathrm{t}, \mathbf{c}), \delta 3.86(3 \mathrm{H}, \mathrm{s}, \mathbf{d}), \delta 4.24-4.17(2 \mathrm{H}, \mathrm{q}, \mathbf{e}), \delta 1.43-1.11(3 \mathrm{H}$, $\mathrm{m}, \mathbf{f})$

anion: $\delta 1.78-1.73(2 \mathrm{H}, \mathrm{t}, \mathbf{g}), \delta 1.43-1.11(14 \mathrm{H}, \mathrm{m}, \mathbf{h}, \mathbf{i}, \mathbf{j}, \mathbf{k}, \mathbf{l}, \mathbf{m}, \mathbf{n}), \delta 0.88-0.83(3 \mathrm{H}, \mathrm{t}, \mathbf{o})$

2-Carboxylate: $\delta 7.65-7.64(1 \mathrm{H}, \mathrm{d}, \mathbf{I}), \delta 7.58-7.57(1 \mathrm{H}, \mathrm{d}, \mathbf{I I}), \delta 3.95(3 \mathrm{H}, \mathrm{s}, \mathbf{I I I}), \delta 4.50-4.42(2 \mathrm{H}, \mathrm{q}, \mathbf{I V}), \delta 1.43-1.11(3 \mathrm{H}, \mathrm{m}, \mathbf{V})$

Figure S9. ${ }^{1} \mathrm{H}$ NMR spectrum of $\left[\mathrm{C}_{2} \mathrm{mim}\right] \mathrm{C}_{12} \mathrm{H}_{23} \mathrm{O}_{2}$. (300MHz, d6-DMSO)

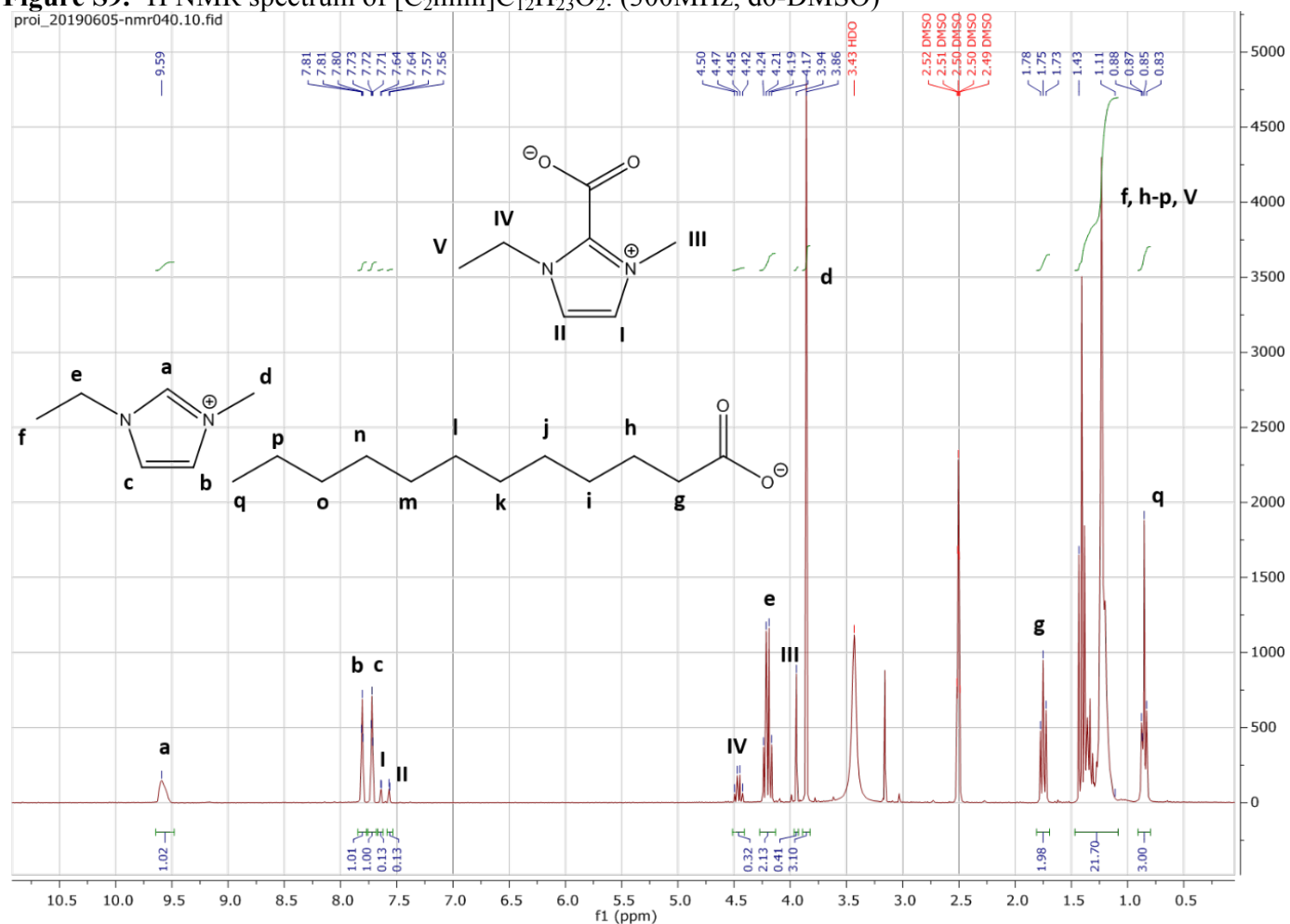

cation: $\delta 9.59(1 \mathrm{H}, \mathrm{s}, \mathbf{a}), \delta 7.81-7.80(1 \mathrm{H}, \mathrm{t}, \mathbf{b}), \delta 7.73-7.71(1 \mathrm{H}, \mathrm{t}, \mathbf{c}), \delta 3.86(3 \mathrm{H}, \mathrm{s}, \mathbf{d}), \delta 4.24-4.17(2 \mathrm{H}, \mathrm{q}, \mathbf{e}), \delta 1.43-1.11(3 \mathrm{H}$, $\mathrm{m}, \mathbf{f})$

anion: $\delta 1.78-1.73(2 \mathrm{H}, \mathrm{t}, \mathbf{g}), \delta 1.43-1.11(18 \mathrm{H}, \mathrm{m}, \mathbf{h}, \mathbf{i}, \mathbf{j}, \mathbf{k}, \mathbf{l}, \mathbf{m}, \mathbf{n}, \mathbf{o}, \mathbf{p}), \delta 0.88-0.83(3 \mathrm{H}, \mathrm{t}, \mathbf{q})$

2-Carboxylate: $\delta 7.64(1 \mathrm{H}, \mathrm{d}, \mathbf{I}), \delta 7.57-7.56(1 \mathrm{H}, \mathrm{d}, \mathbf{I I}), \delta 3.94(3 \mathrm{H}, \mathrm{s}, \mathbf{I I I}), \delta 4.50-4.42(2 \mathrm{H}, \mathrm{q}, \mathbf{I V}), \delta 1.43-1.11(3 \mathrm{H}, \mathrm{m}, \mathbf{V})$ 
Figure S10. ${ }^{1} \mathrm{H}$ NMR spectrum of $\left[\mathrm{C}_{2} \mathrm{mim}\right] \mathrm{C}_{14} \mathrm{H}_{27} \mathrm{O}_{2}$. (300MHz, d6-DMSO)

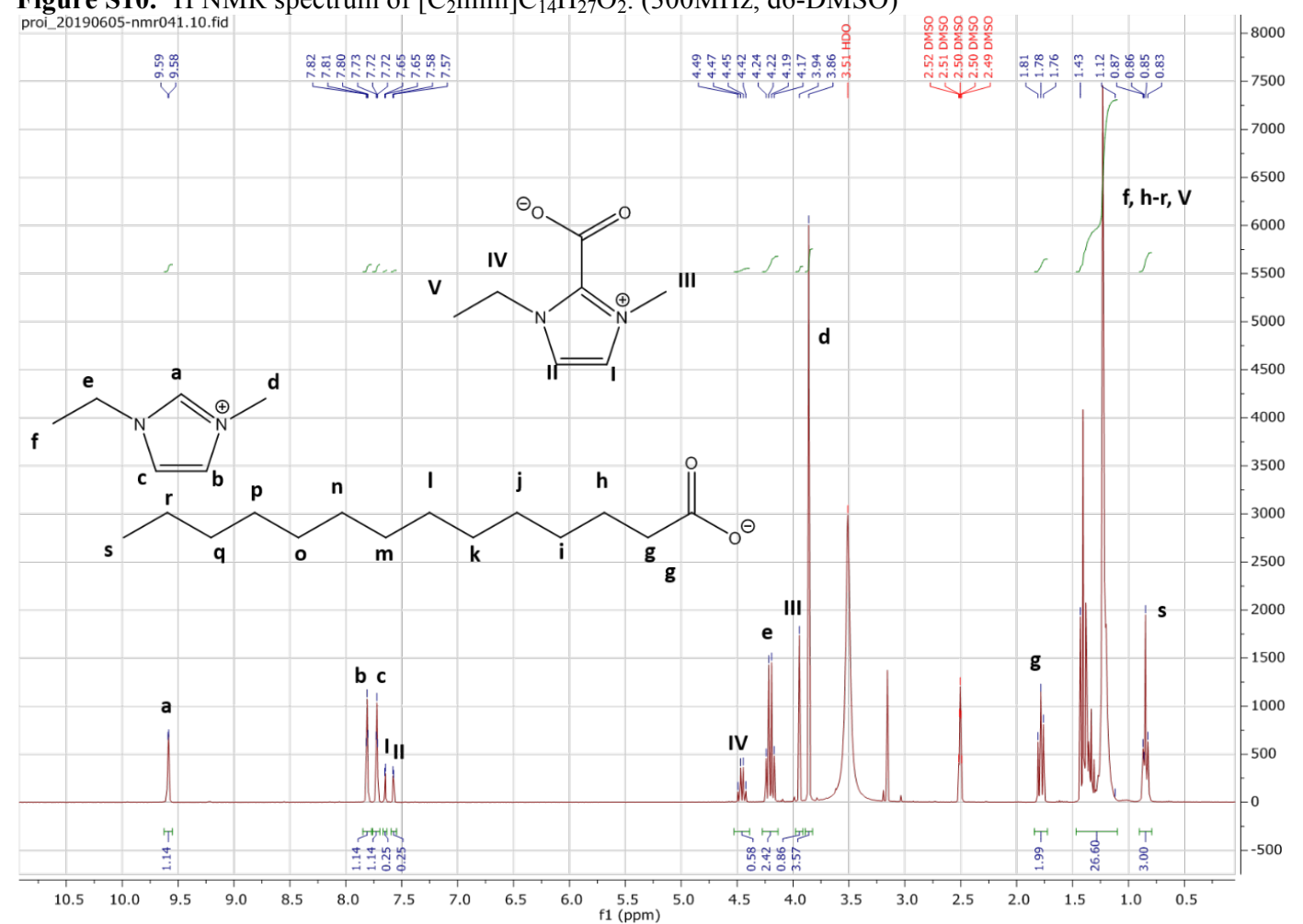

cation: $\delta 9.59-9.58(1 \mathrm{H}, \mathrm{s}, \mathbf{a}), \delta 7.82-7.80(1 \mathrm{H}, \mathrm{t}, \mathbf{b}), \delta 7.73-7.72(1 \mathrm{H}, \mathrm{t}, \mathbf{c}), \delta 3.86(3 \mathrm{H}, \mathrm{s}, \mathbf{d}), \delta 4.24-4.17(2 \mathrm{H}, \mathrm{q}, \mathbf{e}), \delta 1.43-1.12$ $(3 \mathrm{H}, \mathrm{m}, \mathbf{f})$

anion: $\delta 1.81-1.76(2 \mathrm{H}, \mathrm{t}, \mathbf{g}), \delta 1.43-1.12(22 \mathrm{H}, \mathrm{m}, \mathbf{h}, \mathbf{i}, \mathbf{j}, \mathbf{k}, \mathbf{l}, \mathbf{m}, \mathbf{n}, \mathbf{o}, \mathbf{p}, \mathbf{q}, \mathbf{r}), \delta 0.87-0.83(3 \mathrm{H}, \mathrm{t}, \mathbf{s})$

2-Carboxylate: $\delta 7.66-7.65(1 \mathrm{H}, \mathrm{d}, \mathbf{I}), \delta 7.58-7.57(1 \mathrm{H}, \mathrm{d}, \mathbf{I I}), \delta 3.94(3 \mathrm{H}, \mathrm{s}, \mathbf{I I I}), \delta 4.49-4.42(2 \mathrm{H}, \mathrm{q}, \mathbf{I V}), \delta 1.43-1.12(3 \mathrm{H}, \mathrm{m}, \mathbf{V})$

Figure S11. ${ }^{1} \mathrm{H}$ NMR spectrum of $\left[\mathrm{C}_{2} \mathrm{mim}\right] \mathrm{C}_{16} \mathrm{H}_{31} \mathrm{O}_{2}$. (300MHz, d6-DMSO)

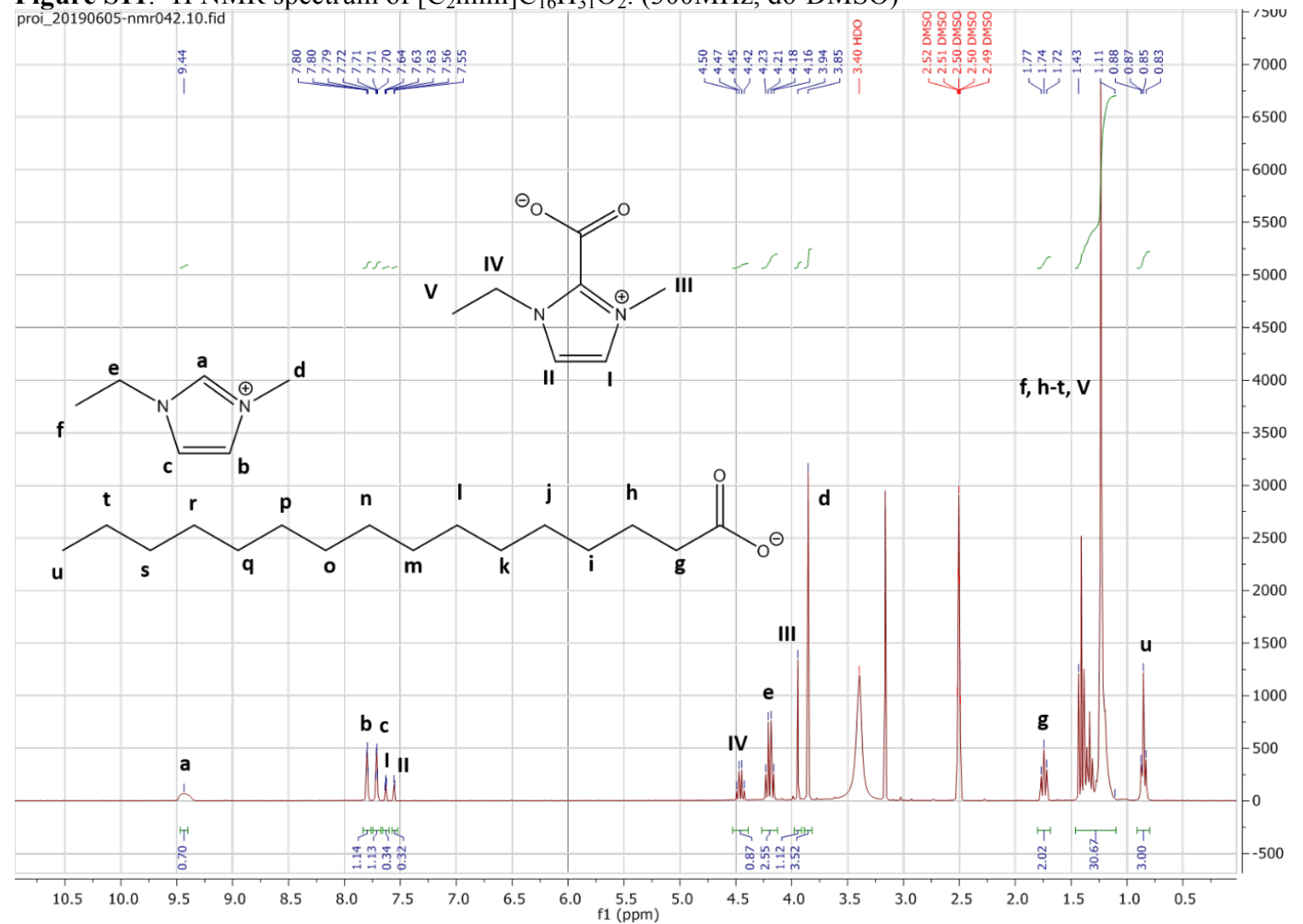

cation: $\delta 9.44(1 \mathrm{H}, \mathrm{s}, \mathbf{a}), \delta 7.80-7.79(1 \mathrm{H}, \mathrm{t}, \mathbf{b}), \delta 7.72-7.71(1 \mathrm{H}, \mathrm{t}, \mathbf{c}), \delta 3.85(3 \mathrm{H}, \mathrm{s}, \mathbf{d}), \delta 4.23-4.16(2 \mathrm{H}, \mathrm{q}, \mathbf{e}), \delta 1.43-1.11(3 \mathrm{H}$, $\mathrm{m}, \mathbf{f})$

anion: $\delta 1.77-1.72(2 \mathrm{H}, \mathrm{t}, \mathbf{g}), \delta 1.43-1.11(26 \mathrm{H}, \mathrm{m}, \mathbf{h}, \mathbf{i}, \mathbf{j}, \mathbf{k}, \mathbf{l}, \mathbf{m}, \mathbf{n}, \mathbf{o}, \mathbf{p}, \mathbf{q}, \mathbf{r}, \mathbf{s}, \mathbf{t}), \delta 0.88-0.83(3 \mathrm{H}, \mathrm{t}, \mathbf{u})$

2-Carboxylate: $\delta 7.64-7.63(1 \mathrm{H}, \mathrm{d}, \mathbf{I}), \delta 7.56-7.55(1 \mathrm{H}, \mathrm{d}, \mathbf{I I}), \delta 3.94(3 \mathrm{H}, \mathrm{s}, \mathbf{I I I}), \delta 4.50-4.42(2 \mathrm{H}, \mathrm{q}, \mathbf{I V}), \delta 1.43-1.11(3 \mathrm{H}, \mathrm{m}, \mathbf{V})$ 
Figure S12. ${ }^{1} \mathrm{H}$ NMR spectrum of $\left[\mathrm{C}_{2} \mathrm{mim}\right] \mathrm{C}_{18} \mathrm{H}_{35} \mathrm{O}_{2}$. (300MHz, d6-DMSO)

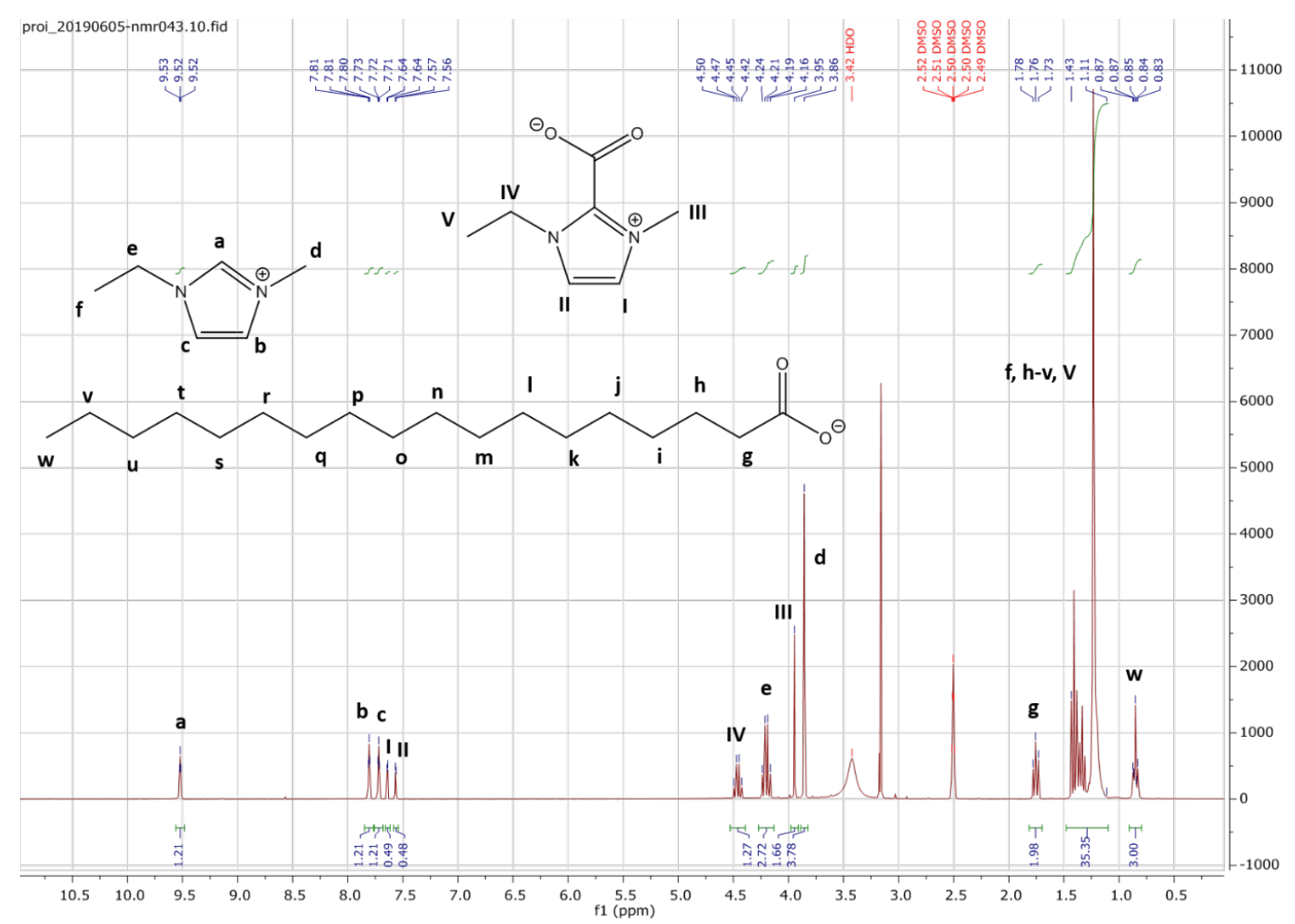

cation: $\delta 9.53-9.52(1 \mathrm{H}, \mathrm{s}, \mathbf{a}), \delta 7.81-7.80(1 \mathrm{H}, \mathrm{t}, \mathbf{b}), \delta 7.73-7.71(1 \mathrm{H}, \mathrm{t}, \mathbf{c}), \delta 3.86(3 \mathrm{H}, \mathrm{s}, \mathbf{d}), \delta 4.24-4.16(2 \mathrm{H}, \mathrm{q}, \mathbf{e}), \delta 1.43-1.11$ $(3 \mathrm{H}, \mathrm{m}, \mathbf{f})$

anion: $\delta 1.78-1.73(2 \mathrm{H}, \mathrm{t}, \mathbf{g}), \delta 1.43-1.11(30 \mathrm{H}, \mathrm{m}, \mathbf{h}, \mathbf{i}, \mathbf{j}, \mathbf{k}, \mathbf{l}, \mathbf{m}, \mathbf{n}, \mathbf{o}, \mathbf{p}, \mathbf{q}, \mathbf{r}, \mathbf{s}, \mathbf{t}, \mathbf{u}, \mathbf{v}), \delta 0.87-0.83(3 \mathrm{H}, \mathrm{t}, \mathbf{w})$

2-Carboxylate: $\delta 7.64(1 \mathrm{H}, \mathrm{d}, \mathbf{I}), \delta 7.57-7.56(1 \mathrm{H}, \mathrm{d}, \mathbf{I I}), \delta 3.95(3 \mathrm{H}, \mathrm{s}, \mathbf{I I I}), \delta 4.50-4.42(2 \mathrm{H}, \mathrm{q}, \mathbf{I V}), \delta 1.43-1.11(3 \mathrm{H}, \mathrm{m}, \mathbf{V})$

Table S4. Calculated and measured mass of [ $C_{2}$ mim] based FAILs. Liquid Chromatography-Mass Spectrometry (LC-MS) was conducted using Electrospray Ionization. The samples dissolved in $\mathrm{MeOH}$ were directly injected with a flow rate of 20 $\mu L / m i n$ with a concentration of $0.1 \mathrm{mg} / \mathrm{mL}$ and measured in positive and negative mode for the analysis of the cations and anions.

\begin{tabular}{|c|c|c|c|c|c|c|}
\hline Cation & Anion & $\mathrm{c}[\mathrm{mg} / \mathrm{ml}]$ & $\begin{array}{c}\mathrm{m} / \mathrm{z} \text { calc. } \\
{\left[\mathrm{C}_{2} \mathrm{mim}^{+}\right.}\end{array}$ & $\begin{array}{c}\mathrm{m} / \mathrm{z} \text { positive } \\
\text { mode } \\
{\left[\mathrm{C}_{2} \mathrm{mim}\right]^{+}}\end{array}$ & $\mathrm{m} / \mathrm{z}$ calc. $\mathrm{FA}^{-}$ & $\begin{array}{l}\mathrm{m} / \mathrm{z} \text { negative } \\
\text { mode } \mathrm{FA}^{-}\end{array}$ \\
\hline$\left[\mathrm{C}_{2} \mathrm{mim}\right]$ & {$\left[\mathrm{C}_{1} \mathrm{H}_{1} \mathrm{O}_{2}\right]$} & 0.0958 & 111.1 & 111.0 & 45.0 & n.s. \\
\hline$\left[\mathrm{C}_{2} \mathrm{mim}\right]$ & {$\left[\mathrm{C}_{2} \mathrm{H}_{3} \mathrm{O}_{2}\right]$} & 0.1014 & 111.1 & 111.0 & 59.0 & 59.0 \\
\hline$\left[\mathrm{C}_{2} \mathrm{mim}\right]$ & {$\left[\mathrm{C}_{3} \mathrm{H}_{5} \mathrm{O}_{2}\right]$} & 0.1034 & 111.1 & 111.0 & 73.0 & 73.0 \\
\hline$\left[\mathrm{C}_{2} \mathrm{mim}\right]$ & {$\left[\mathrm{C}_{4} \mathrm{H}_{7} \mathrm{O}_{2}\right]$} & 0.1058 & 111.1 & 111.0 & 87.1 & 87.0 \\
\hline$\left[\mathrm{C}_{2} \mathrm{mim}\right]$ & {$\left[\mathrm{C}_{6} \mathrm{H}_{11} \mathrm{O}_{2}\right]$} & 0.1020 & 111.1 & 111.0 & 115.1 & 115.1 \\
\hline$\left[\mathrm{C}_{2} \mathrm{mim}\right]$ & {$\left[\mathrm{C}_{8} \mathrm{H}_{15} \mathrm{O}_{2}\right]$} & 0.0928 & 111.1 & 111.0 & 143.1 & 143.2 \\
\hline$\left[\mathrm{C}_{2} \mathrm{mim}\right]$ & {$\left[\mathrm{C}_{9} \mathrm{H}_{17} \mathrm{O}_{2}\right]$} & 0.0968 & 111.1 & 111.0 & 157.1 & 157.2 \\
\hline$\left[\mathrm{C}_{2} \mathrm{mim}\right]$ & {$\left[\mathrm{C}_{10} \mathrm{H}_{19} \mathrm{O}_{2}\right]$} & 0.1008 & 111.1 & 111.0 & 171.1 & 171.3 \\
\hline$\left[\mathrm{C}_{2} \mathrm{mim}\right]$ & {$\left[\mathrm{C}_{12} \mathrm{H}_{23} \mathrm{O}_{2}\right]$} & 0.0972 & 111.1 & 111.0 & 199.2 & 199.3 \\
\hline$\left[\mathrm{C}_{2} \mathrm{mim}\right]$ & {$\left[\mathrm{C}_{14} \mathrm{H}_{27} \mathrm{O}_{2}\right]$} & 0.0986 & 111.1 & 111.0 & 227.2 & 227.4 \\
\hline$\left[\mathrm{C}_{2} \mathrm{mim}\right]$ & {$\left[\mathrm{C}_{16} \mathrm{H}_{31} \mathrm{O}_{2}\right]$} & 0.1138 & 111.1 & 111.0 & 255.2 & 255.5 \\
\hline$\left[\mathrm{C}_{2} \mathrm{mim}\right]$ & {$\left[\mathrm{C}_{18} \mathrm{H}_{35} \mathrm{O}_{2}\right]$} & 0.1011 & 111.1 & 111.0 & 283.3 & 283.6 \\
\hline
\end{tabular}

n.s. no signal as $\mathrm{m} / \mathrm{z}$ is below 50 


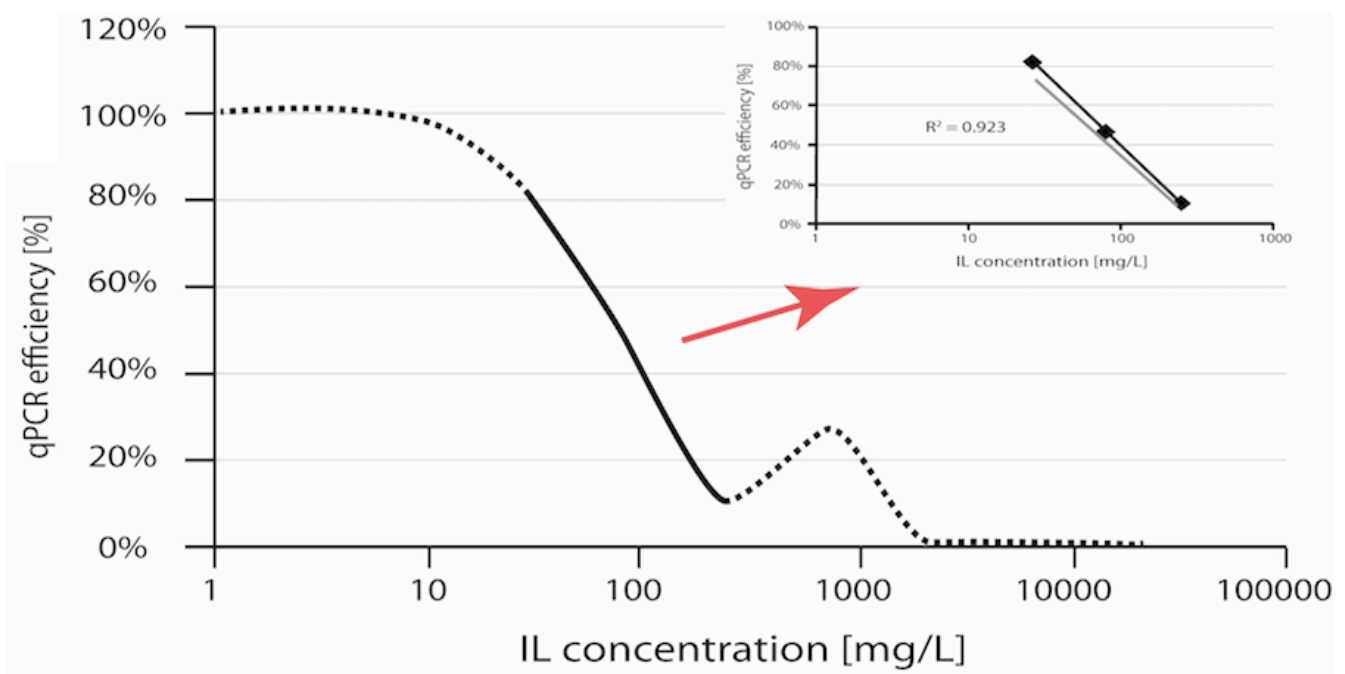

Figure S13. Depiction of an enzymatic inhibition by an IL and calculation of the $E C_{50}$ value by linear regression (indicated by a red arrow).

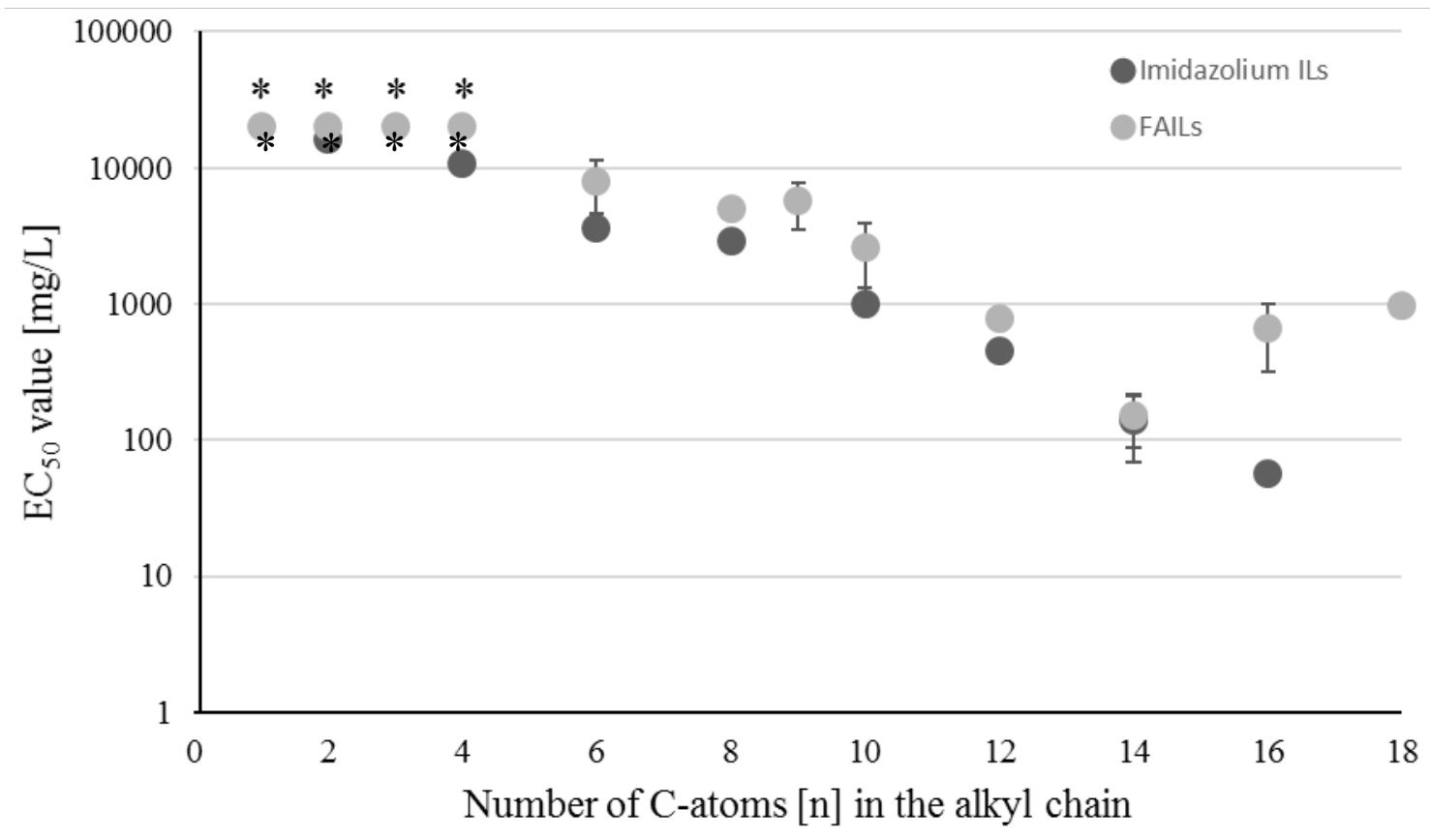

Figure S14. $\mathrm{EC}_{50}$ values $[\mathrm{mg} / \mathrm{L}]$ of FAILs $\left(\left[\mathrm{C}_{2} \mathrm{mim}\right] \mathrm{C}_{\mathrm{n}} \mathrm{H}_{\left(\mathrm{n}^{*} 2\right)-1} \mathrm{O}_{2}\right)$ investigated in this study with increasing anionic alkyl chain lengths and imidazolium-based ILs $\left(\left[\mathrm{C}_{\mathrm{n}} \mathrm{mim}\right] \mathrm{Cl}\right)$ with increasing cationic alkyl chain lengths. * indicates $\mathrm{EC}_{50}$ values above the experimental detection limit. 
Table S5. Virucidal concentration [mg/L] including upper and lower limit of imidazolium-based ILs with increasing cationic alkyl side-chain lengths and FAILs with increasing anionic alkyl side-chain lengths.

\begin{tabular}{|c|c|c|c|c|c|}
\hline \multirow[t]{2}{*}{ Imidazolium-based ILs } & & \multicolumn{4}{|l|}{ Fatty acid-based IL } \\
\hline & Phi6 $^{\text {b }}$ & & $\mathrm{P} 100^{\mathrm{c}}$ & $\mathrm{MS} 2^{\mathrm{c}}$ & Phi6 $^{\mathrm{c}}$ \\
\hline \multirow[t]{2}{*}[\mathrm{C}_{1}\mathrm{mim}]{$\mathrm{Cl}$} & $>50,000$ & {$\left[\mathrm{C}_{2} \mathrm{mim}\right] \mathrm{CH}_{1} \mathrm{O}_{2}$} & $>50,000$ & $>50,000$ & $>50,000$ \\
\hline & {$[>50,000 ;>50,000]$} & & {$[>50,000 ;>50,000]$} & {$[>50,000 ;>50,000]$} & {$[>50,000 ;>50,000]$} \\
\hline \multirow[t]{2}{*}[\mathrm{C}_{2}\mathrm{mim}]{$\mathrm{Cl}$} & $>50,000$ & {$\left[\mathrm{C}_{2} \operatorname{mim}\right] \mathrm{C}_{2} \mathrm{H}_{3} \mathrm{O}_{2}$} & $>50,000$ & $>50,000$ & 25,000 \\
\hline & {$[>50,000 ;>50,000]$} & & {$[>50,000 ;>50,000]$} & {$[>50,000 ;>50,000]$} & {$[25,000 ; 25,000]$} \\
\hline \multirow[t]{2}{*}[\mathrm{C}_{4}\mathrm{mim}]{$\mathrm{Cl}$} & $>50,000$ & {$\left[\mathrm{C}_{2} \mathrm{mim}\right] \mathrm{C}_{3} \mathrm{H}_{5} \mathrm{O}_{2}$} & $>50,000$ & $>50,000$ & $>50,000$ \\
\hline & {$[>50,000 ;>50,000]$} & & {$[>50,000 ;>50,000]$} & {$[>50,000 ;>50,000]$} & {$[>50,000 ;>50,000]$} \\
\hline \multirow[t]{2}{*}[\mathrm{C}_{6}\mathrm{mim}]{$\mathrm{Cl}$} & 50,000 & {$\left[\mathrm{C}_{2} \mathrm{mim}\right] \mathrm{C}_{4} \mathrm{H}_{7} \mathrm{O}_{2}$} & $>50,000$ & $>50,000$ & $>50,000$ \\
\hline & {$[50,000 ; 50,000]$} & & {$[>50,000 ;>50,000]$} & {$[>50,000 ;>50,000]$} & {$[>50,000 ;>50,000]$} \\
\hline \multirow[t]{2}{*}[\mathrm{C}_{8}\mathrm{mim}]{$\mathrm{Cl}$} & 25,000 & {$\left[\mathrm{C}_{2} \operatorname{mim}\right] \mathrm{C}_{6} \mathrm{H}_{11} \mathrm{O}_{2}$} & $>50,000$ & $>50,000$ & 50,000 \\
\hline & {$[25,000 ; 25,000]$} & & {$[>50,000 ;>50,000]$} & {$[>50,000 ;>50,000]$} & {$[50,000 ; 50,000]$} \\
\hline \multirow[t]{2}{*}[\mathrm{C}_{10}\mathrm{mim}]{$\mathrm{Cl}$} & 10,000 & {$\left[\mathrm{C}_{2} \mathrm{mim}\right] \mathrm{C}_{8} \mathrm{H}_{15} \mathrm{O}_{2}$} & $>50,000$ & $>50,000$ & 50,000 \\
\hline & {$[10,000 ; 10,000]$} & & {$[>50,000 ;>50,000]$} & {$[>50,000 ;>50,000]$} & {$[50,000 ; 50,000]$} \\
\hline \multirow[t]{2}{*}[\mathrm{C}_{12}\mathrm{mim}]{$\mathrm{Cl}$} & 100 & {$\left[\mathrm{C}_{2} \operatorname{mim}\right] \mathrm{C}_{9} \mathrm{H}_{17} \mathrm{O}_{2}$} & $>50,000$ & $>50,000$ & 50,000 \\
\hline & {$[100 ; 100]$} & & {$[>50,000 ;>50,000]$} & {$[>50,000 ;>50,000]$} & {$[50,000 ; 50,000]$} \\
\hline \multirow[t]{2}{*}[\mathrm{C}_{14}\mathrm{mim}]{$\mathrm{Cl}$} & 100 & {$\left[\mathrm{C}_{2} \mathrm{mim}\right] \mathrm{C}_{10} \mathrm{H}_{19} \mathrm{O}_{2}$} & 50,000 & $>50,000$ & 10,000 \\
\hline & {$[100 ; 100]$} & & {$[>50,000 ; 50,000]$} & {$[>50,000 ;>50,000]$} & {$[10,000 ; 10,000]$} \\
\hline \multirow[t]{8}{*}[\mathrm{C}_{16}\mathrm{mim}]{$\mathrm{Cl}$} & 100 & {$\left[\mathrm{C}_{2} \mathrm{mim}\right] \mathrm{C}_{12} \mathrm{H}_{23} \mathrm{O}_{9}$} & 15,000 & $>50,000$ & 10,000 \\
\hline & {$[100 ; 100]$} & & {$[25,000 ; 10,000]$} & $(>50.000 ;>50.000)$ & {$[10,000 ; 10,000]$} \\
\hline & & {$\left[\mathrm{C}_{2} \mathrm{mim}\right] \mathrm{C}_{14} \mathrm{H}_{27} \mathrm{O}_{2}$} & 18,571 & $>50,000$ & 10,000 \\
\hline & & & {$[25,000 ; 10,000]$} & {$[>50,000 ;>50,000]$} & {$[10,000 ; 10,000]$} \\
\hline & & {$\left[\mathrm{C}_{2} \mathrm{mim}\right] \mathrm{C}_{16} \mathrm{H}_{31} \mathrm{O}_{2}$} & 10,000 & $>50,000$ & 22,000 \\
\hline & & & {$[10,000 ; 10,000]$} & {$[>50,000 ;>50,000]$} & {$[25,000 ; 10,000]$} \\
\hline & & {$\left[\mathrm{C}_{2} \mathrm{mim}\right] \mathrm{C}_{18} \mathrm{H}_{35} \mathrm{O}_{2}$} & 20,000 & $>50,000$ & 20,000 \\
\hline & & & {$[25,000 ; 10,000]$} & {$[>50,000 ;>50,000]$} & {$[25,000 ; 10,000]$} \\
\hline
\end{tabular}

${ }^{\mathrm{b}}$ Values for virucidal concentration were taken from the study by Sommer et al. $2018^{31}$.

'All values for the virucidal concentration were obtained in this study through experimentation

Table S6. Virucidal concentration $\left[\mathrm{mM}^{1}, \mathrm{mg} / \mathrm{L}^{2}\right]$ including upper and lower limit of imidazolium-based ILs with increasing cationic alkyl side-chain lengths and FAILs with increasing anionic alkyl side-chain lengths.

\begin{tabular}{|c|c|c|c|c|c|c|}
\hline & P100 & MS2 & Phi6 & P100 & MS2 & Phi6 \\
\hline $\mathrm{CH}_{2} \mathrm{O}_{2}$ & $\begin{array}{c}2.17^{1} \\
{[2.17 ; 2.17]}\end{array}$ & $\begin{array}{c}>1086.25 \\
{[>1086.25 ;>1086.25]}\end{array}$ & $\begin{array}{c}21.72 \\
{[21.72 ; 21.72]}\end{array}$ & $\begin{array}{c}100^{2} \\
{[100 ; 100]}\end{array}$ & $\begin{array}{c}>50.000 \\
{[>50.000 ;>50.000]}\end{array}$ & $\begin{array}{c}1000 \\
{[1000 ; 1000]}\end{array}$ \\
\hline $\mathrm{C}_{2} \mathrm{H}_{4} \mathrm{O}_{2}$ & $\begin{array}{c}16.65 \\
{[16.65 ; 16.65]}\end{array}$ & $\begin{array}{c}>832.64 \\
{[>832.64 ;>832.64]}\end{array}$ & $\begin{array}{c}166.52 \\
{[166.52 ; 166.52]}\end{array}$ & $\begin{array}{c}1000 \\
{[1000 ; 1000]}\end{array}$ & $\begin{array}{c}>50.000 \\
{[>50.000 ;>50.000]}\end{array}$ & $\begin{array}{c}10000 \\
{[10000 ; 10000]}\end{array}$ \\
\hline $\mathrm{C}_{3} \mathrm{H}_{6} \mathrm{O}_{2}$ & $\begin{array}{c}104.62 \\
{[1.35 ; 13.5]}\end{array}$ & $\begin{array}{c}>674.95 \\
{[>674.95 ;>674.95]}\end{array}$ & $\begin{array}{c}134.99 \\
{[134.99 ; 134.99]}\end{array}$ & $\begin{array}{c}7750 \\
{[1000 ; 100]}\end{array}$ & $\begin{array}{c}>50.000 \\
{[>50.000 ;>50.000]}\end{array}$ & $\begin{array}{c}10000 \\
{[10.000 ; 10.000]}\end{array}$ \\
\hline $\mathrm{C}_{4} \mathrm{H}_{8} \mathrm{O}_{2}$ & $\begin{array}{c}87.96 \\
{[1.13 ; 11.35]}\end{array}$ & $\begin{array}{c}>567.47 \\
{[>567.47 ;>567.47]}\end{array}$ & $\begin{array}{c}113.49 \\
{[113.49 ; 113.49]}\end{array}$ & $\begin{array}{c}7750 \\
{[1000 ; 100]}\end{array}$ & $\begin{array}{c}>50.000 \\
{[>50.000 ;>50.000]}\end{array}$ & $\begin{array}{c}10000 \\
{[10.000 ; 10.000]}\end{array}$ \\
\hline $\mathrm{C}_{6} \mathrm{H}_{12} \mathrm{O}_{2}$ & $\begin{array}{c}86.09 \\
{[86.09 ; 86.09]}\end{array}$ & $\begin{array}{c}>430.44 \\
{[>430.44 ;>430.44]}\end{array}$ & $\begin{array}{c}86.09 \\
{[86.09 ; 86.09]}\end{array}$ & $\begin{array}{c}10000 \\
{[10.000 ; 10.000]}\end{array}$ & $\begin{array}{c}>50.000 \\
{[>50.000 ;>50.000]}\end{array}$ & $\begin{array}{c}10000 \\
{[10.000 ; 10.000]}\end{array}$ \\
\hline $\mathrm{C}_{8} \mathrm{H}_{16} \mathrm{O}_{2}$ & $\begin{array}{c}346.72 \\
{[>346.72 ; 346.72]}\end{array}$ & $\begin{array}{c}>346.72 \\
{[>346.72 ;>346.72]}\end{array}$ & $\begin{array}{c}346.72 \\
{[346.72 ; 346.72]}\end{array}$ & $\begin{array}{c}50000 \\
{[>50.000 ; 50.000]}\end{array}$ & $\begin{array}{c}>50.000 \\
{[>50.000 ;>50.000]}\end{array}$ & $\begin{array}{c}50,000 \\
{[50.000 ; 50.000]}\end{array}$ \\
\hline $\mathrm{C}_{9} \mathrm{H}_{18} \mathrm{O}_{2}$ & $\begin{array}{c}>315.98 \\
{[>315.98 ;>315.98]}\end{array}$ & $\begin{array}{c}>315.98 \\
{[>315.98 ;>315.98]}\end{array}$ & $\begin{array}{c}315.98 \\
{[>315.98 ; 315.98]}\end{array}$ & $\begin{array}{c}>50.000 \\
{[>50.000 ;>50.000]}\end{array}$ & $\begin{array}{c}>50.000 \\
{[>50.000 ;>50.000]}\end{array}$ & $\begin{array}{c}50000 \\
{[>50.000 ; 50.000]}\end{array}$ \\
\hline $\mathrm{C}_{10} \mathrm{H}_{20} \mathrm{O}_{2}$ & $\begin{array}{c}>290.24 \\
{[>290.24>290.24]}\end{array}$ & $\begin{array}{c}>290.24 \\
{[>290.24>290.24]}\end{array}$ & $\begin{array}{c}>290.24 \\
{[>290.24>290.24]}\end{array}$ & $\begin{array}{c}>50.000 \\
{[>50.000 ;>50.000]}\end{array}$ & $\begin{array}{c}>50.000 \\
{[>50.000 ;>50.000]}\end{array}$ & $\begin{array}{c}>50.000 \\
{[>50.000 ;>50.000]}\end{array}$ \\
\hline $\mathrm{C}_{12} \mathrm{H}_{24} \mathrm{O}_{2}$ & $\begin{array}{c}>49.92 \\
{[>49.92 ;>49.92]}\end{array}$ & $\begin{array}{c}>49.92 \\
{[>49.92 ;>49.92]}\end{array}$ & $\begin{array}{c}>49.92 \\
{[>49.92 ;>49.92]}\end{array}$ & $\begin{array}{c}>10.000 \\
{[>10.000 ;>10.000]}\end{array}$ & $\begin{array}{c}>10.000 \\
{[>10.000 ;>10.000]}\end{array}$ & $\begin{array}{c}>10.000 \\
{[>10.000 ;>10.000]}\end{array}$ \\
\hline $\mathrm{C}_{14} \mathrm{H}_{28} \mathrm{O}_{2}$ & $\begin{array}{c}>43.79 \\
{[>43.79 ;>43.79]}\end{array}$ & $\begin{array}{c}>43.79 \\
{[>43.79 ;>43.79]}\end{array}$ & $\begin{array}{c}>43.79 \\
{[>43.79 ;>43.79]}\end{array}$ & $\begin{array}{c}>10.000 \\
{[>10.000 ;>10.000]}\end{array}$ & $\begin{array}{c}>10.000 \\
{[>10.000 ;>10.000]}\end{array}$ & $\begin{array}{c}>10.000 \\
{[>10.000 ;>10.000]}\end{array}$ \\
\hline $\mathrm{C}_{16} \mathrm{H}_{32} \mathrm{O}_{2}$ & $\begin{array}{c}>38.4 \\
{[>38.4 ;>38.4]}\end{array}$ & $\begin{array}{c}>38.4 \\
{[>38.4 ;>38.4]}\end{array}$ & $\begin{array}{c}>38.4 \\
{[>38.4 ;>38.4]}\end{array}$ & $\begin{array}{c}>10.000 \\
{[>10.000 ;>10.000]}\end{array}$ & $\begin{array}{c}>10.000 \\
{[>10.000 ;>10.000]}\end{array}$ & $\begin{array}{c}>10.000 \\
{[>10.000 ;>10.000]}\end{array}$ \\
\hline $\mathrm{C}_{18} \mathrm{H}_{36} \mathrm{O}_{2}$ & $\begin{array}{c}>35.15 \\
{[>35.15 ;>35.15]}\end{array}$ & $\begin{array}{c}>35.15 \\
{[>35.15 ;>35.15]}\end{array}$ & $\begin{array}{c}>35.15 \\
{[>35.15 ;>35.15]}\end{array}$ & $\begin{array}{c}>10.000 \\
{[>10.000 ;>10.000]}\end{array}$ & $\begin{array}{c}>10.000 \\
{[>10.000 ;>10.000]}\end{array}$ & $\begin{array}{c}>10.000 \\
{[>10.000 ;>10.000]}\end{array}$ \\
\hline
\end{tabular}

${ }^{1}$ Virucidal concentration in $\mathrm{mM}$ 
${ }^{2}$ Virucidal concentration in $\mathrm{mg} / \mathrm{L}$ 